\title{
The Beta Weibull-G Family of Distributions: Model, Properties and Application
}

\author{
Boikanyo Makubate ${ }^{1}$, Broderick O. Oluyede ${ }^{2}$, Gofaone Motobetso ${ }^{1}$, Shujiao Huang ${ }^{3}$ \& Adeniyi F. Fagbamigbe ${ }^{1,4}$ \\ ${ }^{1}$ Department of Mathematics and Statistical Sciences, Botswana International University of Science and Technology, BW \\ ${ }^{2}$ Department of Mathematical Sciences, Georgia Southern University, USA \\ ${ }^{3}$ BBVA Compass, Houston, USA \\ ${ }^{4}$ Department of Epidemiology and Medical Statistics, University of Ibadan, NG \\ Correspondence: B. O. Oluyede, Department of Mathematical Sciences, Georgia Southern University, Statesboro, GA, \\ 30460, USA. E-mail: boluyede@ georgiasouthern.edu
}

Received: November 8, 2017 Accepted: December 5, 2017 Online Published: January 18, 2018

doi:10.5539/ijsp.v7n2p12 URL: https://doi.org/10.5539/ijsp.v7n2p12

\begin{abstract}
A new family of generalized distributions called the beta Weibull-G (BWG) distribution is proposed and developed. This new class of distributions has several new and well known distributions including exponentiated-G, Weibull-G, RayleighG, exponential-G, beta exponential-G, beta Rayleigh-G, beta Rayleigh exponential, beta-exponential-exponential, Weibulllog-logistic distributions, as well as several other distributions such as beta Weibull-Uniform, beta Rayleigh-Uniform, beta exponential-Uniform, beta Weibull-log logistic and beta Weibull-exponential distributions as special cases. Series expansion of the density function, hazard function, moments, mean deviations, Lorenz and Bonferroni curves, Rényi entropy, distribution of order statistics and maximum likelihood estimates of the model parameters are given. Application of the model to real data set is presented to illustrate the importance and usefulness of the special case beta Weibull-log-logistic distribution.
\end{abstract}

Keywords: Generalized distribution, Beta Weibull-G distribution, Beta Weibull log-logistic distribution, Exponentiated$\mathrm{G}$ distribution, Maximum Likelihood Estimation

\section{Introduction}

In this note, we present and study a new class of generalized distributions namely the beta Weibull-G family of distributions. This new family of distributions is a mixture of the exponentiated-G family of distributions and it includes new distributions such as the beta-Weibull-uniform (BWU), beta-Weibull exponential (BWE), and beta Weibull log-logistic (BWLLoG) distributions among several other distributions as special cases. The Weibull-G family of distributions generalizes the Weibull distribution which is considered one of the most important distribution used in the modeling lifetime data.

Oluyede, Pu, Makubate \& Qiu (2018) introduced the gamma Weibull-G family of distributions by combining the gammagenerator with the Weibull-G family of distributions which was defined by Bourguignon, Silva \& Cordeiro (2014). This new class of distributions is flexible in accommodating all forms of hazard rate functions and contains several well known and new sub-models such as Weibull, Rayleigh, exponential, modified Weibull, gamma-modified Weibull, gammamodified exponential, gamma-Weibull and gamma-Rayleigh distributions.

Weibull distribution is often the initial choice when modeling monotone hazard rate functions, however, when modeling phenomenon with non-monotone failure rates such as bathtub and unimodal failure rates, it is not a reasonable fit (Almalki and Nadarajah (2015), Elbatal and Merovci (2015)). To avoid this problem, several generalizations of the Weibull distribution have been introduced for modeling complex data and obtaining better fits. Oluyede, Huang \& Yang (2015) developed and presented a new class of generalized modified Weibull distribution in this context. Gurvich, DiBenedetto \& Ranade (1997) developed a new general family of univariate distributions generated from the Weibull distribution for characterizing the random strength of brittle materials. Other generalizations include the gamma-exponentiated Weibull distributions (GEW) in line with the work done by Zografos and Balakrishnan (2009). See Pinho, Cordeiro \& Nobre (2012) and references therein for details.

We are motivated to introduce this new and flexible extended generator, the beta Weibull-G family of distributions by combining the beta generator with the Weibull-G family of distributions given by Bourguignon et al. (2014). We hope this new family of generalized distributions will provide a much better fit in certain practical situations. We also provide 
a rigorous derivation of the statistical properties of the new family of distributions.

This content of this paper is outlined as follows. The Weibull-G family of distributions is given in Section 2 . In Section 3 , some mathematical and statistical properties of the beta Weibull-G family of distributions including the sub-models, expansion of density function, hazard and reverse hazard functions, quantile function, moments, conditional moments, mean deviations, Bonferroni and Lorenz curves, order statistics, Rényi entropy and maximum likelihood estimates are presented. Some examples of the beta Weibull-G distribution are given in Section 4. The special case of the beta Weibull log-logistic distribution is treated in detail in Section 5. A Monte Carlo simulation study to examine the performance of the maximum likelihood estimates of the BWLLoG distribution is presented in Section 6. An application of the special case of the BWLLoG distribution is given in Section 7, followed by concluding remarks in Section 8.

\section{The Weibull-G Family of Distributions}

The Weibull-G family of distributions was proposed by Bourguignon et al. (2014). Considering the cumulative distribution function (cdf) of the Weibull distribution expressed as $F(x)=1-\exp \left\{-\alpha x^{\beta}\right\}$ for $x>0$ with $\alpha$ and $\beta$ as scale and shape parameters, and by replacing $x$ with $G(x ; \xi) / \bar{G}(x ; \xi)$, where $\bar{G}(x ; \xi)=1-G(x ; \xi)$, then the Weibull-G cdf and probability density function (pdf) are given by

$$
F_{W G}(x ; \alpha, \beta, \xi)=1-\exp \left\{-\alpha\left[\frac{G(x ; \xi)}{\bar{G}(x ; \xi)}\right]^{\beta}\right\}
$$

and

$$
f_{W G}(x ; \alpha, \beta, \xi)=\alpha \beta g(x ; \xi) \frac{G(x ; \xi)^{\beta-1}}{\bar{G}(x ; \xi)^{\beta+1}} \exp \left\{-\alpha\left[\frac{G(x ; \xi)}{\bar{G}(x ; \xi)}\right]^{\beta}\right\},
$$

respectively, where $G(x ; \xi)$ and $g(x ; \xi)$ as the cdf and pdf of any baseline distribution depending on a parameter vector $\xi$. Tahir, Zubair, Mansoor, Alizadeh, Cordeiro \& Hamedani (2016) introduced a new Weibull-G (NWG) family of distributions which is flexible since the hazard rate shapes include: constant, increasing, decreasing, bathtub, upside-down bathtub, J, reversed-J and S. The sub-models or special cases include Weibull-Uniform (WU), Weibull-Rayleigh (WR), Weibull-Logistic (WLLoG), Weibull-Burr XII (WBXII) and Weibull-Normal (WN) distributions. The density of the new family of distributions can be symmetrical, left skewed, right skewed, bathtub and reversed J shaped. The cdf and pdf are given as

$$
F_{N W G}(x ; \alpha, \beta, \xi)=1-\exp \left\{-\alpha[-\log [G(x ; \xi)]]^{\beta}\right\}
$$

and

$$
f_{N W G}(x ; \alpha, \beta, \xi)=\alpha \beta \frac{g(x ; \xi)}{G(x ; \xi)}[-\log [G(x ; \xi)]]^{\beta-1} \exp \left\{-\alpha[-\log [G(x ; \xi)]]^{\beta}\right\},
$$

respectively, where $g(x ; \xi)$ is the parent pdf.

\section{The Beta Weibull-G Family of Distributions}

A new generalization of the Weibull-G family of distributions referred to as the beta Weibull-G (BWG) family of distributions is proposed and studied with the aim of attracting wider application in reliability, survival analysis, biology and other areas of research. This generalization contains several new sub-models such as the beta Exponentiated-G (BEG), beta Rayleigh-G (BRG), Lehmann-G, Exponentiated Weibull-G (EWG), Exponentiated Rayleigh-G (ERG), Exponentiated Exponential-G (EEG), Weibull-G (WG), Rayleigh-G (RG), Exponential-G (EG) distributions, along with several others. The proposed BWG distribution seems to be an important distribution that can be used in modeling reliability and survival data due to its flexibility in accommodating all forms of hazard functions. Special cases of BWG distribution are useful for modeling bathtub-shaped failure rate data and testing goodness-of-fit of some special sub-models like the exponential-uniform (EU), exponential-exponential (EE), exponentiated modified Weibull (EMW), and a host of other sub-distributions.

A general class of distributions called the beta- $\mathrm{G}$ family of distributions is defined by

$$
F(x)=\frac{B_{G(x)}(a, b)}{B(a, b)},
$$

where $G(x)$ denote a cdf of a continuous random variable $X, B_{G(x)}(a, b)=\int_{0}^{G(x)} t^{a-1}(1-t)^{b-1} d t$, and $\frac{1}{B(a, b)}=\frac{\Gamma(a+b)}{\Gamma(a) \Gamma(b)}$. The above generalized class of distributions was motivated by the work of Eugene, Famoye \& Lee (2002). The authors studied the beta-normal distribution. Other discussed beta-G distributions include the work by Lee, Famoye \& Olumolade (2007). 
By taking the baseline cdf $G(x)$ in equation (5) to be the cdf of the Gumbel, Frechet, exponential, and exponentiated exponential distributions, Nadarajah and Kotz (2004) proposed beta Gumbel distribution, Nadarajah and Gupta (2004) studied the beta Frechet distribution, Nadarajah and Kotz (2005) proposed beta exponential distribution and Barreto-Souza, Santos \& Cordeiro (2010) developed the beta exponentiated exponential distribution. Pescim, Demetrio, Cordeiro, Ortega \& Urbano (2010) proposed and studied the beta generalized half-normal distribution, with half-normal and generalized half-normal distributions as special cases. Recently, Carrasco, Cordeiro \& Ortega (2008) studied the generalized modified Weibull distribution and more recently, Oluyede and Yang (2015) developed a new class of distributions called the beta generalized Lindley distribution.

Therefore, by choosing $G(x)$ as the Weibull-G distribution, a larger and readily applicable class of distributions is obtained. Recall, the Weibull-G distribution has the pdf given by

$$
f_{W G}(x)=\alpha \beta g(x ; \underline{\theta}) \frac{G(x ; \underline{\theta})^{\beta-1}}{\bar{G}(x ; \underline{\theta})^{\beta+1}} \exp \left\{-\alpha\left[\frac{G(x ; \underline{\theta})}{\bar{G}(x ; \underline{\theta})}\right]^{\beta}\right\}
$$

for $x, \alpha, \beta>0$. The cdf of $\mathrm{BWG}$ distribution is given by

$$
\begin{aligned}
& F_{B W G}(x ; a, b, \alpha, \beta, \underline{\theta})=\frac{1}{B(a, b)} \int_{0}^{1-\exp \left\{-\alpha\left[\frac{G(x, \theta)}{G(x ; \theta)}\right]^{\beta}\right\}} t^{a-1}(1-t)^{b-1} d t
\end{aligned}
$$

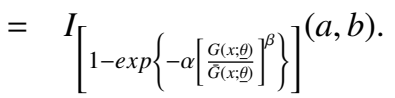

If $|t|<1$ and $b>0$ is a real non-integer, we apply the series representation

$$
(1-t)^{b-1}=\sum_{j=0}^{\infty} \frac{(-1)^{j} \Gamma(b)}{j ! \Gamma(b-j)} t^{j}
$$

to the BWG distribution to obtain

$$
\begin{aligned}
F_{B W G}(x ; a, b, \alpha, \beta, \underline{\theta}) & =\frac{1}{B(a, b)} \sum_{j=0}^{\infty} \frac{(-1)^{j} \Gamma(b)}{j ! \Gamma(b-j)} \int_{0}^{1-\exp \left\{-\alpha\left[\frac{G(x ; \theta)}{\bar{G}(x ; \underline{\theta})}\right]^{\beta}\right\}} t^{a+j-1} d t \\
& =\frac{1}{B(a, b)} \sum_{j=0}^{\infty} \frac{(-1)^{j} \Gamma(b)\left[1-\exp \left\{-\alpha\left[\frac{G(x ; \underline{\theta})}{\bar{G}(x ; \underline{\theta})}\right]^{\beta}\right\}\right]^{a+j}}{j !(a+j) \Gamma(b-j)}
\end{aligned}
$$

where $B(a, b)=\int_{0}^{\infty} t^{a-1}(1-t)^{b-1} d t=\frac{\Gamma(a) \Gamma(b)}{\Gamma(a+b)}$ and $a>0, b>0$. The pdf of BWG distribution is given by

$$
f_{B W G}(x ; a, b, \alpha, \beta, \underline{\theta})=\frac{\alpha \beta}{B(a, b)} g(x ; \underline{\theta}) \frac{G(x ; \underline{\theta})^{\beta-1}}{\bar{G}(x ; \underline{\theta})^{\beta+1}} \exp \left\{-\alpha b\left[\frac{G(x ; \underline{\theta})}{\bar{G}(x ; \underline{\theta})}\right]^{\beta}\right\}\left[1-\exp \left\{-\alpha\left[\frac{G(x ; \underline{\theta})}{\bar{G}(x ; \underline{\theta})}\right]^{\beta}\right\}\right]^{a-1},
$$

for $a, b, \alpha, \beta>0$. In order to avoid issues or problems with over parametrization, only cases in which the parent distribution $G(x ; \underline{\theta})$ has one parameter and cases in which the Weibull distribution of the BWG model is replaced by the exponential distribution leading to the beta exponential-G distribution are considered. Several sub-models can be obtained when the parameter vector $\underline{\theta}$ is changed and some of these sub-models or special cases are presented in Table 3.1 below:

Table 1. Some Sub-Models of the BWG Family of Distributions

\begin{tabular}{ccc}
\hline Distribution & $G(x ; \underline{\theta}) /[1-G(x ; \underline{\theta})]$ & $\underline{\theta}$ \\
\hline Uniform $(0<x<\theta)$ & $x /(\theta-x)$ & $\theta$ \\
Exponential $(x>0)$ & $e^{\lambda x}-1$ & $\lambda$ \\
Weibull $(x>0)$ & $e^{\lambda x^{\gamma}}-1$ & $(\lambda, \gamma)$ \\
Fréchet $(x>0)$ & $\left(e^{\lambda x^{\gamma}}-1\right)^{-1}$ & $(\lambda, \gamma)$ \\
Half-logistic $(x>0)$ & $\left(e^{x}-1\right) / 2$ & $\emptyset$ \\
Power function $(0<x<1 / \theta)$ & {$\left[(\theta x)^{-k}-1\right]^{-1}$} & $(\theta, k)$ \\
Pareto $(x \geq \theta)$ & $(x / \theta)^{k}-1$ & $(\theta, k)$ \\
Log-logistic $(x>0)$ & $(x / s)^{c}$ & $(s, c)$ \\
\hline
\end{tabular}




\subsection{Some Sub-Models}

1. When $\beta=1$, we have the beta exponential-G (BEG) distribution with pdf given as

$$
f_{B E G}(x ; a, b, \alpha, \underline{\theta})=\frac{\alpha}{B(a, b)} g(x ; \underline{\theta}) \frac{1}{\bar{G}(x ; \underline{\theta})^{2}} \exp \left\{-\alpha b\left[\frac{G(x ; \underline{\theta})}{\bar{G}(x ; \underline{\theta})}\right]\right\}\left[1-\exp \left\{-\alpha\left[\frac{G(x ; \underline{\theta})}{\bar{G}(x ; \underline{\theta})}\right]\right\}\right]^{a-1} .
$$

2. When $\beta=2$, the resulting distribution is the beta Rayleigh-G (BRG) distribution with pdf given by

$$
f_{B R G}(x ; a, b, \alpha, \underline{\theta})=\frac{2 \alpha}{B(a, b)} g(x ; \underline{\theta}) \frac{G(x ; \underline{\theta})}{\bar{G}(x ; \underline{\theta})^{3}} \exp \left\{-\alpha b\left[\frac{G(x ; \underline{\theta})}{\bar{G}(x ; \underline{\theta})}\right]^{2}\right\}\left[1-\exp \left\{-\alpha\left[\frac{G(x ; \underline{\theta})}{\bar{G}(x ; \underline{\theta})}\right]^{2}\right\}\right]^{a-1} .
$$

3. If $a=1$, we obtain the Lehmann-G distribution, where the pdf is given by

$$
f_{\text {Lehmann }}(x ; b, \alpha, \beta, \underline{\theta})=b \alpha \beta \frac{G(x ; \underline{\theta})^{\beta-1}}{\bar{G}(x ; \underline{\theta})^{\beta+1}} g(x ; \underline{\theta})\left[\exp \left\{-\alpha\left[\frac{G(x ; \underline{\theta})}{\bar{G}(x ; \underline{\theta})}\right]^{\beta}\right\}\right]^{b-1} .
$$

4. Setting $b=1$, we obtain the exponentiated Weibull-G (EWG) distribution. The EWG pdf is

$$
f_{E W G}(x ; a, \alpha, \beta, \underline{\theta})=\alpha \beta g(x ; \underline{\theta}) \frac{G(x ; \underline{\theta})^{\beta-1}}{\bar{G}\left(x ; \underline{\theta}^{\beta+1}\right.}\left[\exp \left\{-\alpha\left[\frac{G(x ; \underline{\theta})}{\bar{G}(x ; \underline{\theta})}\right]^{\beta}\right\}\right] a\left[1-\exp \left\{-\alpha\left[\frac{G(x ; \underline{\theta})}{\bar{G}(x ; \underline{\theta})}\right]^{\beta}\right\}\right]^{a-1} .
$$

5. When $a=1$ and $\beta=2$, we obtain the exponentiated Rayleigh-G (ERG) distribution with the pdf

$$
f_{E R G}(x ; \alpha, b, \underline{\theta})=2 \alpha g(x ; \underline{\theta}) \frac{G(x ; \underline{\theta})}{\bar{G}(x ; \underline{\theta})^{3}} \exp \left\{-\alpha\left[\frac{G(x ; \underline{\theta})}{\bar{G}(x ; \underline{\theta})}\right]^{2}\right\} b\left[\exp \left\{-\alpha\left[\frac{G(x ; \underline{\theta})}{\bar{G}(x ; \underline{\theta})}\right]^{2}\right\}\right]^{b-1} .
$$

6. When $a=\beta=1$, we obtain the exponentiated exponential-G (EEG) distribution, where the pdf is given by

$$
f_{E E G}(x ; \alpha, b, \underline{\theta})=\alpha g(x ; \underline{\theta}) \frac{1}{\bar{G}(x ; \underline{\theta})^{2}} \exp \left\{-\alpha\left[\frac{G(x ; \underline{\theta})}{\bar{G}(x ; \underline{\theta})}\right]\right\} b\left[1-\exp \left\{-\alpha\left[\frac{G(x ; \underline{\theta})}{\bar{G}(x ; \underline{\theta})}\right]\right\}\right]^{b-1} .
$$

7. When $a=b=1$, we obtain the Weibull-G (WG) distribution (Bourguignon et al. (2014)). The WG pdf is given by

$$
f_{W G}(x ; \alpha, \beta, \underline{\theta})=\alpha \beta g(x ; \underline{\theta}) \frac{G(x ; \underline{\theta})^{\beta-1}}{\bar{G}\left(x ; \underline{\theta}^{\beta+1}\right.} \exp \left\{-\alpha\left[\frac{G(x ; \underline{\theta})}{\bar{G}(x ; \underline{\theta})}\right]^{\beta}\right\} \text {. }
$$

8. When $a=b=1$ and $\beta=2$, we obtain the Rayleigh-G (RG) distribution, where the pdf is given by

$$
f_{R G}(x ; \alpha, \underline{\theta})=2 \alpha g(x ; \underline{\theta}) \frac{G(x ; \underline{\theta})}{\bar{G}(x ; \underline{\theta})^{3}} \exp \left\{-\alpha\left[\frac{G(x ; \underline{\theta})}{\bar{G}(x ; \underline{\theta})}\right]^{2}\right\} .
$$

9. When $a=b=\beta=1$, we obtain the exponential-G (EG) distribution, where the pdf is given by

$$
f_{E G}(x ; \alpha, \underline{\theta})=\alpha g(x ; \underline{\theta}) \frac{G(x ; \underline{\theta})}{\bar{G}(x ; \underline{\theta})^{2}} \exp \left\{-\alpha\left[\frac{G(x ; \underline{\theta})}{\bar{G}(x ; \underline{\theta})}\right]\right\} .
$$

\subsection{Expansion of the BWG Density Function}

Considering the binomial series expansion given by

$$
(1-z)^{b-1}=\sum_{i=0}^{\infty} \frac{(-1)^{i} \Gamma(b)}{i ! \Gamma(b-i)} z^{i},
$$


for $b>0$ and $|z|<1$, we have

$$
\left[1-\exp \left\{-\alpha\left[\frac{G(x ; \underline{\theta})}{\bar{G}(x ; \underline{\theta})}\right]^{\beta}\right\}\right]^{a-1}=\sum_{i=0}^{\infty} \frac{(-1)^{i} \Gamma(a)}{i ! \Gamma(a-i)}\left[\exp \left\{-\alpha i\left[\frac{G(x ; \underline{\theta})}{\bar{G}(x ; \underline{\theta})}\right]^{\beta}\right\}\right] .
$$

Inserting this into the pdf of the BWG distribution, we obtain

$$
f_{B W G}(x)=\frac{\alpha \beta}{B(a, b)} g(x ; \underline{\theta}) \frac{G(x ; \underline{\theta})^{\beta-1}}{\bar{G}(x ; \underline{\theta})^{\beta+1}} \sum_{i=0}^{\infty} \frac{(-1)^{i} \Gamma(a)}{i ! \Gamma(a-i)}\left[\exp \left\{-\alpha(b+i)\left[\frac{G(x ; \underline{\theta})}{\bar{G}(x ; \underline{\theta})}\right]^{\beta}\right\}\right] .
$$

Considering the series expansion of the exponential function below

$$
\exp \left\{-\alpha(b+i)\left[\frac{G(x ; \underline{\theta})}{\bar{G}(x ; \underline{\theta})}\right]^{\beta}\right\}=\sum_{j=0}^{\infty} \frac{(-1)^{j}[\alpha(b+i)]^{j}}{j !}\left[\frac{G(x ; \underline{\theta})}{\bar{G}(x \underline{\theta})}\right]^{\beta j},
$$

and inserting equation (10) into the pdf of the BWG distribution, we obtain

$$
\begin{aligned}
f_{B W G}(x) & =\frac{\alpha \beta}{B(a, b)} g(x ; \underline{\theta}) \frac{G(x ; \underline{\theta})^{\beta-1}}{\bar{G}(x ; \underline{\theta})^{\beta+1}} \sum_{i, j=0}^{\infty} \frac{(-1)^{i+j}[\alpha(b+i)]^{j} \Gamma(a)}{i ! j ! \Gamma(a-i)} \frac{G(x ; \underline{\theta})^{\beta j}}{\bar{G}(x ; \underline{\theta})^{\beta j}} \\
& =\frac{\alpha \beta}{B(a, b)} g(x ; \underline{\theta}) \sum_{i, j=0}^{\infty} \frac{(-1)^{i+j}[\alpha(b+i)]^{j} \Gamma(a)}{i ! j ! \Gamma(a-i)} \frac{G(x ; \underline{\theta})^{\beta j+\beta-1}}{\bar{G}(x ; \underline{\theta})^{\beta j+\beta+1}} .
\end{aligned}
$$

Note that

$$
[\bar{G}(x ; \underline{\theta})]^{-(\beta j+\beta+1)}=\sum_{k=0}^{\infty} \frac{\Gamma(\beta j+\beta+k+1)}{k ! \Gamma(\beta j+\beta+1)}[G(x ; \underline{\theta})]^{k},
$$

so that the BWG pdf can be written as:

$$
\begin{aligned}
f_{B W G}(x) & =\frac{\alpha \beta}{B(a, b)} \sum_{i, j, k=0}^{\infty} \frac{(-1)^{i+j}[\alpha(b+i)]^{j} \Gamma(a) \Gamma(\beta j+\beta+k+1)}{i ! j ! k ! \Gamma(a-i) \Gamma(\beta j+\beta+1)} g(x ; \underline{\theta})[G(x ; \underline{\theta})]^{\beta j+\beta+k-1} \\
& =\sum_{i, j, k=0}^{\infty} \frac{\alpha \beta}{B(a, b)} \frac{(-1)^{i+j}[\alpha(b+i)]^{j} \Gamma(a) \Gamma(\beta j+\beta+k+1)}{i ! j ! k ! \Gamma(a-i) \Gamma(\beta j+\beta+1)(\beta j+\beta+k)}(\beta j+\beta+k) g(x ; \underline{\theta})[G(x ; \underline{\theta})]^{\beta j+\beta+k-1} \\
& =\sum_{i, j, k=0}^{\infty} w^{*}(i, j, k)(\beta j+\beta+k) g(x ; \underline{\theta})[G(x ; \underline{\theta})]^{\beta j+\beta+k-1}
\end{aligned}
$$

where

$$
w^{*}(i, j, k)=\frac{\alpha \beta}{B(a, b)} \frac{(-1)^{i+j}[\alpha(b+i)]^{j} \Gamma(a) \Gamma(\beta j+\beta+k+1)}{i ! j ! k ! \Gamma(a-i) \Gamma(\beta j+\beta+1)(\beta j+\beta+k)},
$$

are the weights functions. The BWG pdf can be written as an infinite mixture of the exponentiated-G (EG) densities (Cordeiro, da Cunha \& Ortega, 2013) with power parameter $\beta(1+j)+k>0$ and parameter vector $\underline{\theta}$. It follows therefore that the BWG density is a linear combination of exponential-G (EG) densities. Thus, the mathematical and statistical properties of the BWG distribution can be readily obtained from those of the EG distribution.

\subsection{Hazard and Reverse Hazard Functions}

The hazard and reverse hazard functions of the BWG distribution are given by

$$
\begin{aligned}
& h_{B W G}(x)=\frac{f_{B W G}(x)}{1-F_{B W G}(x)}
\end{aligned}
$$

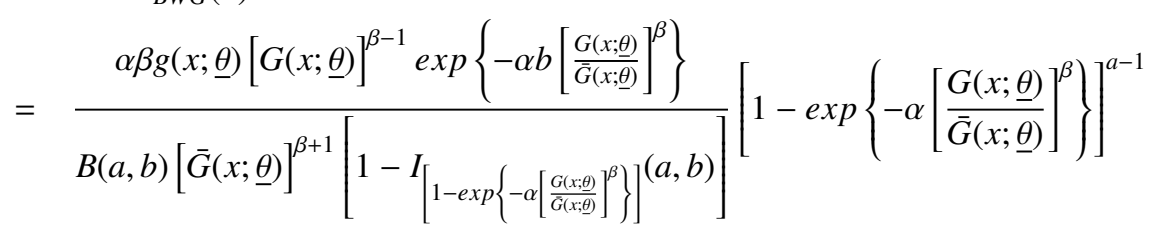


and

$$
\begin{aligned}
& \tau_{B W G}(x)=\frac{f_{B W G}(x)}{F_{B W G}(x)}
\end{aligned}
$$

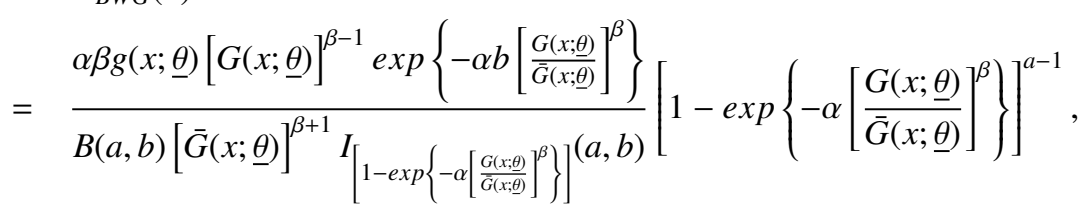

respectively.

\subsection{Quantile Function}

The quantile function of the beta Weibull-G family of distributions is obtained by inverting $F_{B W G}(x)=u$, where $F_{B W G}(x)$ is given by equation (6), and $u$ to be a uniform variate on the interval $[0,1]$. That is, we solve

$$
\left.I_{1-\exp }\left\{-\alpha\left[\frac{G(x, \theta)}{G(x, \theta)}\right]^{\beta}\right\}\right]^{(a, b)=u,}
$$

that is,

$$
\exp \left\{-\alpha\left[\frac{G(x ; \underline{\theta})}{\bar{G}(x ; \underline{\theta})}\right]^{\beta}\right\}=1-I_{u}^{-1}(a, b) .
$$

By taking logarithm on both sides, we have

$$
\frac{G(x ; \underline{\theta})}{\bar{G}(x ; \underline{\theta})}=\left(-\frac{1}{\alpha} \ln \left[1-I_{u}^{-1}(a, b)\right]\right)^{\frac{1}{\beta}},
$$

so that

$$
G(x ; \underline{\theta})=\left(-\frac{1}{\alpha} \ln \left[1-I_{u}^{-1}(a, b)\right]\right)^{\frac{1}{\beta}}-G(x ; \underline{\theta})\left(-\frac{1}{\alpha} \ln \left[1-I_{u}^{-1}(a, b)\right]\right)^{\frac{1}{\beta}},
$$

and

$$
G(x ; \underline{\theta})=\frac{\left(-\frac{1}{\alpha} \ln \left[1-I_{u}^{-1}(a, b)\right]\right)^{\frac{1}{\beta}}}{1+\left(-\frac{1}{\alpha} \ln \left[1-I_{u}^{-1}(a, b)\right]\right)^{\frac{1}{\beta}}}=: q .
$$

Consequently,

$$
x_{q}=G^{-1}\left[\frac{\left(-\frac{1}{\alpha} \ln \left[1-I_{u}^{-1}(a, b)\right]\right)^{\frac{1}{\beta}}}{1+\left(-\frac{1}{\alpha} \ln \left[1-I_{u}^{-1}(a, b)\right]\right)^{\frac{1}{\beta}}}\right],
$$

where $I_{u}^{-1}(a, b)$ is the inverse of the incomplete beta function ratio.

\subsection{Moments, Conditional Moments and Mean Deviations}

There are several characteristics and features of a distribution that can be studied through its moments. Moments are often used to obtain important features of distributions such as measures of central tendency, dispersion, skewness and kurtosis. In this section, we present the moments, conditional moments and mean deviations of the BWG family of distributions.

\subsubsection{Moments}

The $s^{\text {th }}$ moment of the BWG family of distributions can be obtained as follows:

$$
\begin{aligned}
E\left(X^{s}\right) & =\int_{0}^{\infty} x^{s} f_{B W G}(x) d x \\
& =\sum_{i, j, k=0}^{\infty} w^{*}(i, j, k) \int_{0}^{\infty} x^{s}(\beta j+\beta+k) g(x ; \underline{\theta})[G(x ; \underline{\theta})]^{\beta j+\beta+k-1} d x \\
& =\sum_{i, j, k=0}^{\infty} w^{*}(i, j, k) \int_{0}^{\infty} x^{s} f_{\beta j+\beta+k}(x) d x \\
& =\sum_{i, j, k=0}^{\infty} w^{*}(i, j, k) I(\beta j+\beta+k),
\end{aligned}
$$


where $w^{*}(i, j, k)$ is given by equation (13), and $I(\beta j+\beta+k)=\int_{0}^{\infty} x^{s} f_{\beta j+\beta+k}(x) d x$ is the $s^{\text {th }}$ moment of the exponentiated-G (EG) distribution with exponential parameter $\beta j+\beta+k$.

\subsubsection{Conditional Moments}

The $s^{\text {th }}$ conditional moments of the BWG family of distributions is given by

$$
\begin{aligned}
E\left(X^{s} \mid X>t\right) & =\frac{1}{\bar{F}_{B W G}(t)} \int_{t}^{\infty} x^{s} f_{B W G}(x) d x \\
& =\frac{1}{\bar{F}_{B W G}(x)} \sum_{i, j, k=0}^{\infty} w^{*}(i, j, k) I(\beta j+\beta+k, s ; t),
\end{aligned}
$$

where $w^{*}(i, j, k)$ is given by equation (13) and $I(\beta j+\beta+k, s, t)=\int_{t}^{\infty} x^{s} f_{\beta j+\beta+k}(x) d x$ is the $s^{t h}$ incomplete moment of the exponentiated-G (EG) distribution with exponential parameters $\beta j+\beta+k>0$.

\subsubsection{Mean Deviation}

The mean deviation about the mean and the mean deviation about the median have been applied to a wide variety of fields, such as studying of income and property in economics. The mean deviation about the mean and mean deviation about the median are defined as

$$
\delta_{1}(X)=\int_{0}^{\infty}|x-\mu| f_{B W G}(x) d x, \quad \text { and } \quad \delta_{2}(X)=\int_{0}^{\infty}|x-M| f_{B W G}(x) d x,
$$

respectively, where $\mu=E(X)$ and $\mathrm{M}=\operatorname{Median}(\mathrm{X})$ denotes the mean and median. These quantities can be expressed as

$$
\delta_{1}(X)=2 \mu F_{B W G}(\mu)-2 \mu+2 \sum_{i, j, k=0}^{\infty} w^{*}(i, j, k) I(\beta j+\beta+k, 1 ; \mu),
$$

and

$$
\delta_{2}(X)=-\mu+2 \sum_{i, j, k=0}^{\infty} w^{*}(i, j, k) I(\beta j+\beta+k, 1 ; M),
$$

respectively.

\subsubsection{Bonferroni and Lorenz Curves}

Bonferroni and Lorenz curves of the BWG distribution are defined as

$$
B(p)=\frac{1}{p \mu} \int_{q}^{\infty} x f_{B W G}(x) d x, \quad \text { and } \quad L(p)=\frac{1}{\mu} \int_{q}^{\infty} x f_{B W G}(x) d x,
$$

respectively, where

$$
\int_{q}^{\infty} x f_{B W G}(x) d x=\sum_{i, j, k=0}^{\infty} w^{*}(i, j, k) I(\beta j+\beta+k, 1 ; q) .
$$

\subsection{Order Statistics}

In order to derive the distribution of the $i^{\text {th }}$ order statistics from the BWG distribution, let $X_{1}, \ldots \ldots, X_{n}$ to be independent identically distributed (iid) random variables from the BWG pdf, $f_{B W G}(x)$ and $X_{1: n}<\ldots .<X_{n: n}$ to denote the corresponding order statistics. The pdf $f_{i: n}(x)$ of the $i^{t h}$ order statistic is given by

$$
f_{i: n}(x)=\frac{n !}{(i-1) !(n-i) !} f_{B W G}(x)\left[F_{B W G}(x)\right]^{i-1}\left[1-F_{B W G}(x)\right]^{n-i} .
$$

That is,

$$
\begin{aligned}
f_{i: n}(x) & =\sum_{p=0}^{n-i} \frac{(-1)^{p} n !}{(i-1) !(n-i-p) ! p !} f_{B W G}(x)\left[F_{B W G}(x)\right]^{p+i-1} \\
& =\sum_{p=0}^{n-i} \frac{(-1)^{p} n !}{(i-1) !(n-i-p) ! p !} \frac{\alpha \beta}{B(a, b)} g(x ; \underline{\theta}) \frac{G(x ; \underline{\theta})^{\beta-1}}{\bar{G}(x ; \underline{\theta})^{\beta+1}} \\
& \times\left[I_{1-\exp \left\{-\alpha\left[\frac{G(x, \theta)}{G(x, \theta)}\right]^{\beta}\right\}}(a, b)\right]^{p+i-1} \exp \left\{-\alpha b\left[\frac{G(x ; \underline{\theta})}{\bar{G}(x ; \underline{\theta})}\right]^{\beta}\right\}\left[1-\exp \left\{-\alpha\left[\frac{G(x ; \underline{\theta})}{\bar{G}(x ; \underline{\theta})}\right]^{\beta}\right\}\right]^{a-1} .
\end{aligned}
$$


Applying the binomial expansion, we obtain

$$
\left[1-\exp \left\{-\alpha\left[\frac{G(x ; \underline{\theta})}{\bar{G}(x ; \underline{\theta})}\right]^{\beta}\right\}\right]^{a-1}=\sum_{i=0}^{\infty} \frac{(-1)^{i} \Gamma(a)}{i ! \Gamma(a-i)} \exp \left\{-\alpha i\left[\frac{G(x ; \underline{\theta})}{\bar{G}(x ; \underline{\theta})}\right]^{\beta}\right\}
$$

and substituting equation (19) into equation (18), we obtain

$$
\begin{aligned}
f_{i: n}(x) & =\sum_{p=0}^{n-i} \frac{(-1)^{p} n !}{(i-1) !(n-i-p) ! p !} \frac{\alpha \beta}{B(a, b)} g(x ; \underline{\theta}) \frac{G(x ; \underline{\theta})^{\beta-1}}{\bar{G}(x ; \underline{\theta})^{\beta+1}} \\
& \times\left[I_{1-\exp \left\{-\alpha\left[\frac{G(x ; \theta)}{\bar{G}(x, \theta)}\right]^{\beta}\right\}}(a, b)\right]^{p+i-1} \exp \left\{-\alpha b\left[\frac{G(x ; \underline{\theta})}{\bar{G}(x ; \underline{\theta})}\right]^{\beta}\right\} \sum_{i=0}^{\infty} \frac{(-1)^{i} \Gamma(a)}{i ! \Gamma(a-i)} \exp \left\{-\alpha i\left[\frac{G(x ; \underline{\theta})}{\bar{G}(x ; \underline{\theta})}\right]^{\beta}\right\} \\
& =\sum_{p=0}^{n-i} \sum_{i=0}^{\infty} \frac{(-1)^{i} \Gamma(a)}{i ! \Gamma(a-i)} \frac{(-1)^{p} n !}{(i-1) !(n-i-p) ! p !} \frac{\alpha \beta}{B(a, b)} g(x ; \underline{\theta}) \frac{G(x ; \underline{\theta})^{\beta-1}}{\bar{G}(x ; \underline{\theta})^{\beta+1}} \\
& \times\left[I_{1-\exp \left\{-\alpha\left[\frac{G(x ; \theta)}{\bar{G}(x, \theta)}\right]^{\beta}\right\}}(a, b)\right]^{p+i-1} \exp \left\{-\alpha(b+i)\left[\frac{G(x ; \underline{\theta})}{\bar{G}(x ; \underline{\theta})}\right]^{\beta}\right\} .
\end{aligned}
$$

Substituting the exponential series expansion

$$
\exp \left\{-\alpha(b+i)\left[\frac{G(x ; \underline{\theta})}{\bar{G}(x ; \underline{\theta})}\right]^{\beta}\right\}=\sum_{j=0}^{\infty} \frac{(-1)^{j}[\alpha(b+i)]^{j}}{j !}\left[\frac{G(x ; \underline{\theta})}{\bar{G}(x ; \underline{\theta})}\right]^{\beta j}
$$

into (20), we have

$$
f_{i: n}(x)=\sum_{p=0}^{n-i} \sum_{i, j=0}^{\infty} \frac{(-1)^{p} n !}{(i-1) !(n-i-p) ! p !} \frac{(-1)^{i+j}[\alpha(b+i)]^{j} \Gamma(a)}{i ! j ! \Gamma(a-i)} \frac{\alpha \beta}{B(a, b)} g(x ; \underline{\theta}) \frac{G(x ; \underline{\theta})^{\beta j+\beta-1}}{\bar{G}(x ; \underline{\theta})^{\beta j+\beta+1}}\left[I_{1-\exp \left\{-\alpha\left[\frac{G(x, \theta)}{[G(x, \theta)}\right]^{\beta}\right\}}(a, b)\right]^{p+i-1} .
$$

Note that

$$
\bar{G}(x ; \underline{\theta})^{-(\beta j+\beta+1)}=\sum_{k=0}^{\infty} \frac{\Gamma(\beta j+\beta+k+1)}{k ! \Gamma(\beta j+\beta+1)}[G(x ; \underline{\theta})]^{k},
$$

therefore the pdf of the $i^{\text {th }}$ order statistic from the BWG distribution reduces to:

$$
\begin{aligned}
f_{i: n}(x) & =\sum_{p=0}^{n-i} \sum_{i, j, k=0}^{\infty} \frac{(-1)^{p} n !}{(i-1) !(n-i-p) ! p !} \frac{(-1)^{i+j}[\alpha(b+i)]^{j} \Gamma(a)}{i ! j ! \Gamma(a-i)} \\
& \times \frac{\alpha \beta}{B(a, b)} g(x ; \underline{\theta}) G(x ; \underline{\theta})^{\beta j+\beta+k-1}\left[I_{1-\exp \left\{-\alpha\left[\frac{G(x ; \theta \theta}{G(x ; \theta)}\right]^{\beta}\right\}}(a, b)\right]^{p+i-1} \\
& =\sum_{p=0}^{n-i} \sum_{i, j, k=0}^{\infty} w^{*}(i, j, k) \frac{(-1)^{p} n !}{(i-1) !(n-i-p) ! p !} g(x ; \underline{\theta}) G(x ; \underline{\theta})^{\beta j+\beta+k-1}\left[I_{1-\exp \left\{-\alpha\left[\frac{G(x, \theta)}{G(x ; \theta)}\right]^{\beta}\right\}}(a, b)\right]^{p+i-1} \\
& =\sum_{p=0}^{n-i} \sum_{i, j, k=0}^{\infty} w^{*}(i, j, k, p) g(x ; \underline{\theta}) G(x ; \underline{\theta})^{\beta j+\beta+k-1}\left[I_{1-\exp \left\{-\alpha\left[\frac{G(x, \theta)}{G(x ; \theta)}\right]^{\beta}\right\}}(a, b)\right]^{p+i-1},
\end{aligned}
$$

where

$$
w^{*}(i, j, k, p)=\frac{\alpha \beta}{B(a, b)} \frac{(-1)^{i+j+p}[\alpha(b+i)]^{j} \Gamma(a) \Gamma(\beta j+\beta+k+1)}{i ! j ! k ! p !(i-1) !(n-i-p) ! \Gamma(a-i) \Gamma(\beta j+\beta+1)(\beta j+\beta+k)} .
$$

\subsection{Rényi Entropy}

The concept of entropy plays an essential role in information theory. In statistics it is used as indices of diversity and quantify the uncertainty or randomness of a system. In this subsection, we present Rényi entropy for the BWG family of distributions. Rényi entropy is defined by

$$
I_{R}(v)=(1-v)^{-1} \log \left[\int_{-\infty}^{\infty} f^{v}(x) d(x)\right],
$$


for $v>0$ and $v \neq 1$. Note that

$$
f_{B W G}^{v}(x)=\frac{(\alpha \beta)^{v}}{B(a, b)^{v}} g^{v}(x ; \underline{\theta}) \frac{G(x ; \underline{\theta})^{\beta v-v}}{\bar{G}\left(x ; \underline{\theta}^{\beta v+v}\right.} \exp \left\{-\alpha b v\left[\frac{G(x ; \underline{\theta})}{\bar{G}(x ; \underline{\theta})}\right]^{\beta}\right\}\left[1-\exp \left\{-\alpha\left[\frac{G(x ; \underline{\theta})}{\bar{G}(x ; \underline{\theta})}\right]^{\beta}\right\}\right]^{a v-v} .
$$

Now, by using the binomial series expansion

$$
\left[1-\exp \left\{-\alpha\left[\frac{G(x ; \underline{\theta})}{\bar{G}(x ; \underline{\theta})}\right]^{\beta}\right\}\right]^{(a v-v+1)-1}=\sum_{i=0}^{\infty} \frac{(-1)^{i} \Gamma(a v-v+1)}{i ! \Gamma(a v-v+1-i)}\left[\exp \left\{-\alpha i\left[\frac{G(x ; \underline{\theta})}{\bar{G}(x ; \underline{\theta})}\right]^{\beta}\right\}\right],
$$

we have

$$
f_{B W G}^{v}(x)=\frac{(\alpha \beta)^{v}}{B(a, b)^{v}} g^{v}(x ; \underline{\theta}) \frac{G(x ; \underline{\theta})^{\beta v-v}}{\bar{G}\left(x ; \underline{\theta}^{)^{\beta v+v}}\right.} \sum_{i=0}^{\infty} \frac{(-1)^{i} \Gamma(a v-v+1)}{i ! \Gamma(a v-v+1-i)} \exp \left\{-\alpha(b v+i)\left[\frac{G(x ; \underline{\theta})}{\bar{G}(x ; \underline{\theta})}\right]^{\beta}\right\} .
$$

Applying the exponential series expansion

$$
\exp \left\{-\alpha(b v+i)\left[\frac{G(x ; \underline{\theta})}{\bar{G}(x ; \underline{\theta})}\right]^{\beta}\right\}=\sum_{j=0}^{\infty} \frac{(-1)^{j}[\alpha(b v+i)]^{j}}{j !}\left[\frac{G(x ; \underline{\theta})}{\bar{G}(x ; \underline{\theta})}\right]^{\beta j},
$$

and inserting this back into equation (25), we have

$$
\begin{aligned}
f_{B W G}^{v}(x) & =\frac{(\alpha \beta)^{v}}{B(a, b)^{v}} \sum_{i, j, k=0}^{\infty} \frac{(-1)^{i+j}[\alpha(b v+i)]^{j} \Gamma(a v-v+1) \Gamma(\beta j+\beta v+k+v)}{i ! j ! k ! \Gamma(a v-v+1-i) \Gamma(\beta j+\beta v+v)}\left[g(x ; \underline{\theta})[G(x ; \underline{\theta})]^{\frac{\beta j+\beta v+k-v}{v}}\right]^{v} \\
& =\frac{(\alpha \beta)^{v}}{B(a, b)^{v}} \sum_{i, j, k=0}^{\infty} \frac{(-1)^{i+j}[\alpha(b v+i)]^{j} \Gamma(a v-v+1) \Gamma(\beta j+\beta v+k+v)}{i ! j ! k ! \Gamma(a v-v+1-i) \Gamma(\beta j+\beta v+v)} \\
& \times\left[\frac{v}{\beta j+\beta v+k+v-v}\right]\left[\frac{\beta j+\beta v+k+v-v}{v} g(x ; \underline{\theta})[G(x ; \underline{\theta})]^{\frac{\beta j+\beta v+k-v}{v}}\right]^{v}
\end{aligned}
$$

Consequently, the Rényi entropy of the BWG distribution is given by

$$
\begin{aligned}
I_{R}(v) & =(1-v)^{-1} \log \left\{\frac{(\alpha \beta)^{v}}{B(a, b)^{v}} \sum_{i, j, k=0}^{\infty} \frac{(-1)^{i+j}[\alpha(b v+i)]^{j} \Gamma(a v-v+1) \Gamma(\beta j+\beta v+k+v)}{i ! j ! k ! \Gamma(a v-v+1-i) \Gamma(\beta j+\beta v+v)}\right. \\
& \left.\times\left[\frac{v}{\beta j+\beta v+k+v-v}\right]^{v} \exp \left((1-v) I_{R_{E G}}(v)\right)\right\}
\end{aligned}
$$

where $v \neq 1, v>0, I_{R_{E G}}(v)$ denote Rényi entropy of the exponentiated-G (EG) distribution with parameters $\beta^{*}=\frac{\beta j+\beta v+k}{v}$ and $\underline{\theta}$. It follows therefore that Rényi entropy of the exponentiated-G distribution can be used to obtain that of the BWG distribution.

\subsection{Maximum Likelihood Estimation}

In this section, the estimates of the parameters of the BWG family of distributions are presented through the method of maximum likelihood estimation (MLE). Let $\Delta=(a, b, \alpha, \beta, \underline{\theta})^{T}$ be the parameter vector and $x=\left(x_{1}, \ldots \ldots, x_{n}\right)^{T}$ be a random sample from the BWG distribution, then the log-likelihood function for $\Delta$ can be written as

$$
\begin{aligned}
\ell(\Delta) & =n[\ln (\alpha)+\ln (\beta)-\ln (B(a, b))]+\sum_{i=0}^{n} \ln \left(g\left(x_{i} ; \underline{\theta}\right)\right)+(\beta-1) \sum_{i=0}^{n} \ln \left(G\left(x_{i} ; \underline{\theta}\right)\right)-(\beta+1) \sum_{i=0}^{n} \ln \left(\bar{G}\left(x_{i} ; \underline{\theta}\right)\right) \\
& -\alpha b \sum_{i=0}^{n}\left[\frac{G\left(x_{i} ; \underline{\theta}\right)}{\bar{G}\left(x_{i} ; \underline{\theta}\right)}\right]^{\beta}+(a-1) \sum_{i=0}^{n} \ln \left[1-\exp \left\{-\alpha\left[\frac{G\left(x_{i} ; \underline{\theta}\right)}{\bar{G}\left(x_{i} ; \underline{\theta}\right)}\right]^{\beta}\right\}\right] .
\end{aligned}
$$

The elements of the score vector, that is, the partial derivatives of $\ell(\Delta)$ with respect to the parameters are

$$
\frac{\partial \ell}{\partial a}=-n[\psi(a)-\psi(a+b)]+\sum_{i=0}^{n} \ln \left[1-\exp \left\{-\alpha\left[\frac{G\left(x_{i} ; \underline{\theta}\right)}{\bar{G}\left(x_{i} ; \underline{\theta}\right)}\right]^{\beta}\right\}\right],
$$




$$
\begin{gathered}
\frac{\partial \ell}{\partial b}=-n[\psi(b)-\psi(a+b)]-\alpha \sum_{i=0}^{n}\left[\frac{G\left(x_{i} ; \underline{\theta}\right)}{\bar{G}\left(x_{i} ; \underline{\theta}\right)}\right]^{\beta}, \\
\frac{\partial \ell}{\partial \alpha}=\frac{n}{\alpha}-b \sum_{i=0}^{n}\left[\frac{G\left(x_{i} ; \underline{\theta}\right)}{\bar{G}\left(x_{i} ; \underline{\theta}\right)}\right]^{\beta}+(a-1) \sum_{i=0}^{n}\left[1-\exp \left\{-\alpha\left[\frac{G\left(x_{i} ; \underline{\theta}\right)}{\bar{G}\left(x_{i} ; \underline{\theta}\right)}\right]^{\beta}\right\}\right]^{-1} \exp \left\{-\alpha\left[\frac{G\left(x_{i} ; \underline{\theta}\right)}{\bar{G}\left(x_{i} ; \underline{\theta}\right)}\right]^{\beta}\right\}\left[\frac{G\left(x_{i} ; \underline{\theta}\right)}{\bar{G}\left(x_{i} ; \underline{\theta}\right)}\right]^{\beta}, \\
\frac{\partial \ell}{\partial \beta}=\frac{n}{\beta}+\sum_{i=0}^{n} \ln \left(G\left(x_{i} ; \underline{\theta}\right)\right)-\sum_{i=0}^{n} \ln \left(\bar{G}\left(x_{i} ; \underline{\theta}\right)\right)-\alpha b \sum_{i=0}^{n}\left[\frac{G\left(x_{i} ; \underline{\theta}\right)}{\bar{G}\left(x_{i} ; \underline{\theta}\right)}\right]^{\beta} \\
\times \ln \left[\frac{G\left(x_{i} ; \underline{\theta}\right)}{\bar{G}\left(x_{i} ; \underline{\theta}\right)}\right]+(a-1) \alpha \sum_{i=0}^{n}\left[1-\exp \left\{-\alpha\left[\frac{G\left(x_{i} ; \underline{\theta}\right)}{\bar{G}\left(x_{i} ; \underline{\theta}\right)}\right]^{\beta}\right\}\right]^{-1} \exp \left\{-\alpha\left[\frac{G\left(x_{i} ; \underline{\theta}\right)}{\bar{G}\left(x_{i} ; \underline{\theta}\right)}\right]^{\beta}\right\}\left[\frac{G\left(x_{i} ; \underline{\theta}\right)}{\bar{G}\left(x_{i} ; \underline{\theta}\right)}\right]^{\beta} \ln \left[\frac{G\left(x_{i} ; \underline{\theta}\right)}{\bar{G}\left(x_{i} ; \underline{\theta}\right)}\right],
\end{gathered}
$$

and

$$
\begin{aligned}
\frac{\partial \ell}{\partial \underline{\theta}_{k}} & =\sum_{i=0}^{n} \frac{1}{g\left(x_{i} ; \underline{\theta}\right)} \frac{\partial}{\partial \underline{\theta}_{k}} g\left(x_{i} ; \underline{\theta}\right)-(\beta-1) \sum_{i=0}^{n} \frac{1}{G\left(x_{i} ; \underline{\theta}\right)} \frac{\partial}{\partial \underline{\theta}_{k}} G\left(x_{i} ; \underline{\theta}\right)-(\beta+1) \sum_{i=0}^{n} \frac{1}{\bar{G}\left(x_{i} ; \underline{\theta}\right)} \frac{\partial}{\partial \underline{\theta}_{k}} \bar{G}\left(x_{i} ; \underline{\theta}\right)-\alpha \beta b \sum_{i=0}^{n}\left[\frac{G\left(x_{i} ; \underline{\theta}\right)}{\bar{G}\left(x_{i} ; \underline{\theta}\right)}\right]^{\beta-1} \\
& \times \frac{\partial}{\partial \underline{\theta}_{k}}\left[\frac{G\left(x_{i} ; \underline{\theta}\right)}{\bar{G}\left(x_{i} ; \underline{\theta}\right)}\right]+(a-1) \alpha \beta \sum_{i=0}^{n}\left[1-\exp \left\{-\alpha\left[\frac{G\left(x_{i} ; \underline{\theta}\right)}{\bar{G}\left(x_{i} ; \underline{\theta}\right)}\right]^{\beta}\right\}\right]^{-1} \exp \left\{-\alpha\left[\frac{G\left(x_{i} ; \underline{\theta}\right)}{\bar{G}\left(x_{i} ; \underline{\theta}\right)}\right]^{\beta}\right\}\left[\frac{G\left(x_{i} ; \underline{\theta}\right)}{\bar{G}\left(x_{i} ; \underline{\theta}\right)}\right]^{\beta-1} \frac{\partial}{\partial \underline{\theta}_{k}}\left[\frac{G\left(x_{i} ; \underline{\theta}\right)}{\bar{G}\left(x_{i} ; \underline{\theta}\right)}\right],
\end{aligned}
$$

respectively, where $\psi(x)$ is the di-gamma function, and $\underline{\theta}_{k}$ is the $k^{\text {th }}$ element of the vector of parameters $\underline{\theta}$.

\section{Some Examples of the BWG Distributions}

We present three special cases of the BWG family of distributions, namely the beta Weibull-Uniform (BWU) distribution, beta Weibull-exponential (BWE) distribution and the beta Weibull-log logistic (BWLLoG) distribution in this section. The BWLLoG distribution is discussed in detail.

\subsection{Beta Weibull-Uniform (BWU) Distribution}

Consider the uniform distribution on the interval $(0, \theta)$ with pdf $g(x)=\frac{1}{\theta}, 0<x<\theta<\infty$ and cdf $G(x)=\frac{x}{\theta}$. The beta Weibull-Uniform (BWU) cdf is given by

$$
\begin{aligned}
F_{B W U}(x ; a, b, \alpha, \beta, \theta) & =\frac{1}{B(a, b)} \int_{0}^{1-\exp \left\{-\alpha\left[\frac{x}{\theta-x}\right]^{\beta}\right\}} t^{a-1}(1-t)^{b-1} d t \\
& =I_{1-\exp \left\{-\alpha\left[\frac{x}{\theta-x}\right]^{\beta}\right\}}(a, b),
\end{aligned}
$$

and the corresponding pdf is

$$
f_{B W U}(x ; a, b, \alpha, \beta, \theta)=\frac{\alpha \beta \theta}{B(a, b)} \frac{x^{\beta-1}}{(\theta-x)^{\beta+1}} \exp \left\{-\alpha b\left[\frac{x}{\theta-x}\right]^{\beta}\right\}\left[1-\exp \left\{-\alpha\left[\frac{x}{\theta-x}\right]^{\beta}\right\}\right]^{a-1},
$$

where $a, b, \alpha, \beta, \theta>0$ and $x \in(0, \theta)$.

The density plot of the BWU distribution is given in Figure 1 which suggests that for some selected values of the parameters, the BWU pdf can be L-shaped or decreasing, and left skewed.

\subsection{Beta Weibull-Exponential (BWE) Distribution}

Consider the exponential distribution with pdf $g(x ; \lambda)=\lambda e^{-\lambda x}$ and $\operatorname{cdf} G(x)=1-e^{-\lambda x}, \lambda>0$. The beta WeibullExponential (BWE) distribution has cdf and pdf given by

$$
\begin{aligned}
F_{B W E}(x ; a, b, \alpha, \beta, \lambda) & =\frac{1}{B(a, b)} \int_{0}^{1-\exp \left\{-\alpha\left[e^{\lambda x}-1\right]^{\beta}\right\}} t^{a-1}(1-t)^{b-1} d t \\
& =I_{1-\exp \left\{-\alpha\left[e^{\lambda x}-1\right]^{\beta}\right\}}(a, b),
\end{aligned}
$$




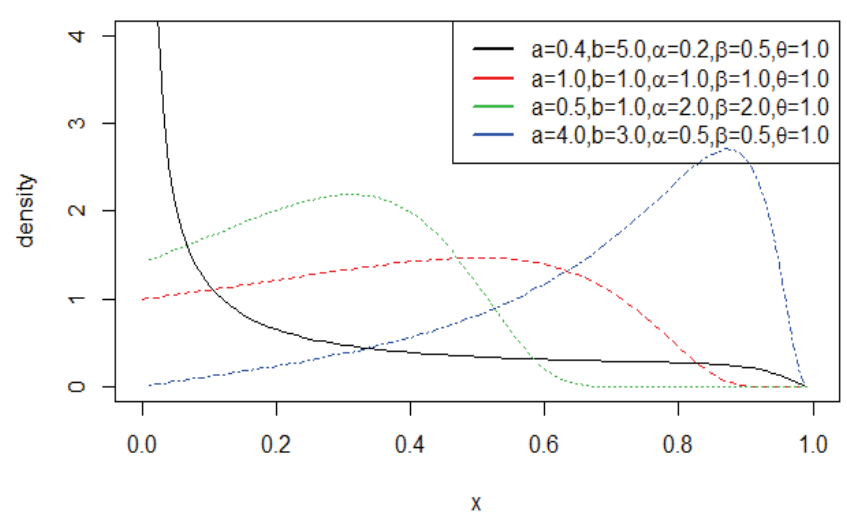

Figure 1. Density plots of the BWU distribution for selected parameter values

and

$$
f_{B W E}(x ; a, b, \alpha, \beta, \lambda)=\frac{\alpha \beta \lambda}{B(a, b)}\left(1-e^{-\lambda x}\right)^{\beta-1} \exp \left\{-\alpha b\left[e^{\lambda x}-1\right]^{\beta}+\lambda \beta x\right\}\left[1-\exp \left\{-\alpha\left[e^{\lambda x}-1\right]^{\beta}\right\}\right]^{a-1}
$$

with $a, b, \alpha, \beta, \lambda>0$ and $x>0$, respectively. The plots of the pdf for some selected parameter values is given in Figure 2 . The plots suggests that the shape of the pdf of the BWE distribution can be L-shaped or right skewed.

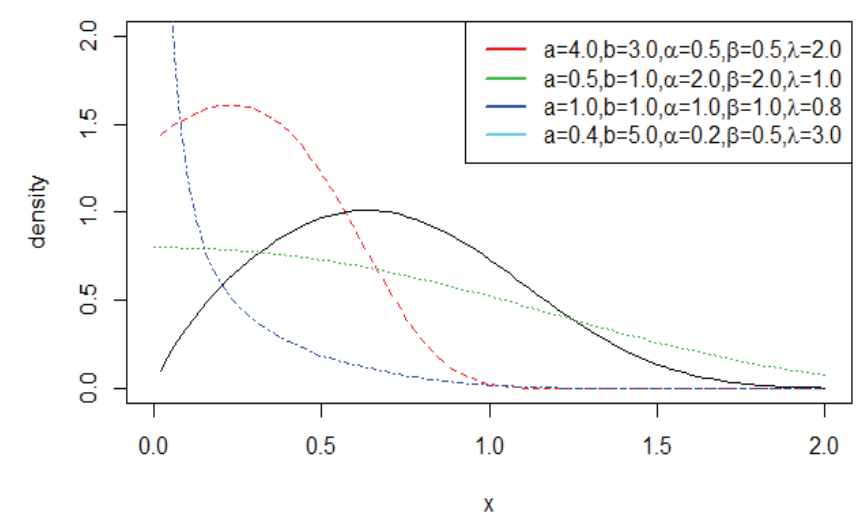

Figure 2. Density plots of the BWE distribution for selected parameter values

\section{Beta Weibull-log logistic (BWLLoG) Distribution}

Consider a log-logistic random variable $X$ with pdf $g(x ; c)=c x^{c-1}\left(1+x^{c}\right)^{-2}$ and $c d f G(x ; c)=\left[1-\left(1+x^{c}\right)^{-1}\right], c>0$. The cdf of the beta Weibull-log logistic (BWLLoG) distribution is given by

$$
\begin{aligned}
F_{B W L L o G}(x ; a, b, c, \alpha, \beta) & =\frac{1}{B(a, b)} \int_{0}^{1-\exp \left\{-\alpha\left[x^{c}\right]^{\beta}\right\}} t^{a-1}(1-t)^{b-1} d t \\
& =I_{1-\exp \left\{-\alpha x^{\alpha \beta}\right\}}(a, b) .
\end{aligned}
$$

for $a, b, c, \alpha, \beta>0$ and $x>0$. The corresponding BWLLoG pdf is

$$
f_{B W L L o G}(x ; a, b, c, \alpha, \beta)=\frac{\alpha \beta c}{B(a, b)} x^{c-1}\left(1+x^{c}\right)^{-2} \frac{\left[1-\left(1+x^{c}\right)^{-1}\right]^{\beta-1}}{\left[\left(1+x^{c}\right)^{-1}\right]^{\beta+1}} \exp \left\{-\alpha b x^{c \beta}\right\}\left[1-\exp \left\{-\alpha x^{c \beta}\right\}\right]^{a-1}
$$


for $a, b, c, \alpha, \beta>0$ and $x>0$. The density plot of the BWLLoG distribution for some selected parameter values is given in Figure 3. The plot shows that the BWLLoG pdf can be L-shaped, left skewed or right skewed depending on the values of the selected parameters.

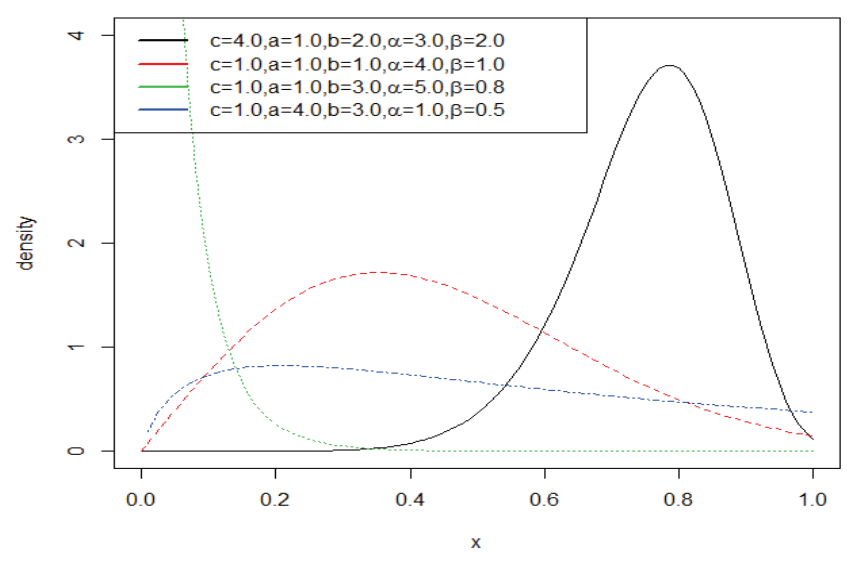

Figure 3. Density plot for the BWLLoG distribution for selected parameter values

\subsection{Some Sub-Models of the BWLLoG Distribution}

Some sub-models of the BWLLoG distribution are presented in this subsection.

1. When $\beta=1$, we obtain the beta exponential-log logistic (BELLoG) distribution. The pdf is given as

$$
f_{B E L L o G}(x ; c, a, b, \alpha)=\frac{\alpha c}{B(a, b)} x^{c-1} \exp \left\{-\alpha b x^{c}\right\}\left[1-\exp \left\{-\alpha x^{c}\right\}\right]^{a-1} .
$$

2. When $b=1$, we obtain the exponentiated Weibull-log logistic (EWLLoG) distribution. The pdf is given as

$$
f_{E W L L o G}(x ; c, a, \alpha, \beta)=\alpha \beta c x^{c-1}\left(1+x^{c}\right)^{-2} \frac{\left[1-\left(1+x^{c}\right)^{-1}\right]^{\beta-1}}{\left[\left(1+x^{c}\right)^{-1}\right]^{\beta+1}} \exp \left\{-\alpha x^{c \beta}\right\}\left[1-\exp \left\{-\alpha x^{c \beta}\right\}\right]^{a-1} .
$$

3. When $a=b=1$, we obtain the Weibull-log logistic (WLLoG) distribution. The pdf is given as

$$
f_{W L L o G}(x ; c, \alpha, \beta)=\alpha \beta c x^{c-1}\left(1+x^{c}\right)^{-2} \frac{\left[1-\left(1+x^{c}\right)^{-1}\right]^{\beta-1}}{\left[\left(1+x^{c}\right)^{-1}\right]^{\beta+1}} \exp \left\{-\alpha x^{c \beta}\right\} .
$$

4. When $b=\beta=1$, we obtain the exponentiated exponential-log logistic (EELLoG) distribution. The pdf is

$$
f_{\text {EELLoG }}(x ; c, a, \alpha)=\alpha c x^{c-1} \exp \left\{-\alpha x^{c}\right\}\left[1-\exp \left\{-\alpha x^{c}\right\}\right]^{a-1} .
$$

\subsection{Expansion of the BWLLoG Density Function}

From the derived expansion of the BWG distribution given in equation (12), the expansion of the BWLLoG distribution is given as

$$
f_{B W L L o G}(x)=\sum_{i, j, k=0}^{\infty} w^{*}(i, j, k)(\beta j+\beta+k) c x^{c-1}\left(1+x^{c}\right)^{-2}\left[1-\left(1+x^{c}\right)^{-1}\right]^{\beta j+\beta+k-1},
$$


where $w^{*}(i, j, k)$ is given in equation (13).

\subsection{Hazard and Reverse Hazard Functions}

The hazard and reverse hazard functions of the BWLLoG distribution are given by

$$
h_{B W L L o G}(x)=\frac{\alpha \beta x^{c-1}\left[1-\left(1+x^{c}\right)^{-1}\right]^{\beta-1} \exp \left\{-\alpha b x^{c \beta}\right\}}{B(a, b)\left[\left(1+x^{c}\right)^{-1}\right]^{\beta+1}\left[1-I_{1-\exp \left\{-\alpha x^{c \beta}\right.}(a, b)\right]}\left[1-\exp \left\{-\alpha x^{c \beta}\right\}\right]^{a-1},
$$

and

$$
\tau_{B W L L o G}(x)=\frac{\alpha \beta x^{c-1}\left[1-\left(1+x^{c}\right)^{-1}\right]^{\beta-1} \exp \left\{-\alpha b x^{c \beta}\right\}}{B(a, b)\left[\left(1+x^{c}\right)^{-1}\right]^{\beta+1}\left[I_{1-\exp \left\{-\alpha x^{c \beta}\right\}}(a, b)\right]}\left[1-\exp \left\{-\alpha x^{c \beta}\right\}\right]^{a-1},
$$

respectively. The plot of the BWLLoG hazard function for selected parameter values is given in Figure 4 . The plots exhibit unimodal, decreasing and upside down bathtub shapes.

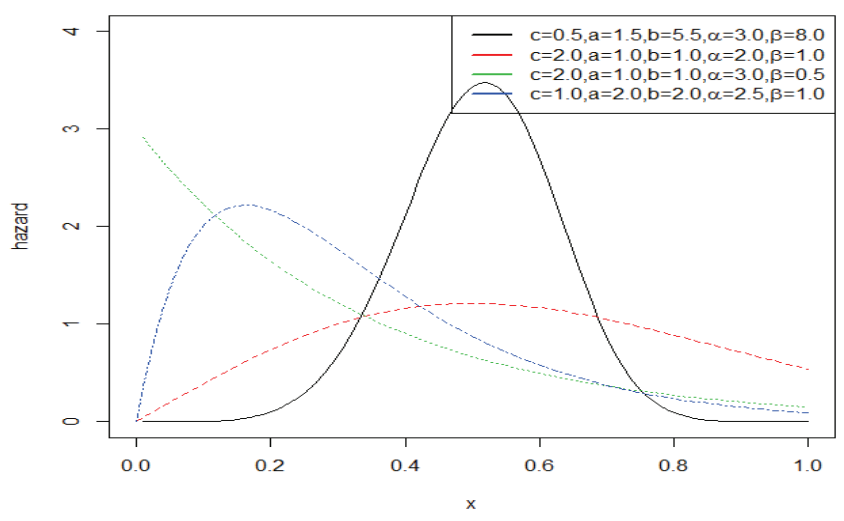

Figure 4. The hazard plot of the BWLLoG distribution for some selected parameter values

\subsection{Quantile Function}

From the quantile function of the BWG distribution given in equation (15), the quantile function of the BWLLoG distribution is obtained from the solution of

$$
-\alpha x^{c \beta}-\log \left[1-I_{u}^{-1}(a, b)\right]=0 .
$$

Table 2 lists the quantiles of the BWLLoG distribution for selected values of the parameters $(a, b, c, \alpha, \beta)$.

Table 2. The BWLLoG quantiles for some selected model parameter values

\begin{tabular}{rrrrr}
\hline \multicolumn{5}{c}{$(a, b, c, \alpha, \beta)$} \\
\cline { 2 - 5 }$u$ & $(1.0,2.0,4.0,3.0,2.0)$ & $(1.0,1.0,2.0,4.0,1.0)$ & $(1.0,3.0,1.0,5.0,0.8)$ & $(4.0,3.0,1.0,1.0,0.5)$ \\
\hline 0.1 & 0.3061057 & 0.1622961 & 0.008780043 & 0.8105134 \\
0.2 & 0.3692741 & 0.2361854 & 0.018595296 & 1.0709395 \\
0.3 & 0.4152169 & 0.2986107 & 0.029722912 & 1.2927493 \\
0.4 & 0.4542297 & 0.3573601 & 0.042568802 & 1.5067108 \\
0.5 & 0.4902414 & 0.4162502 & 0.057762265 & 1.7283114 \\
0.6 & 0.5256638 & 0.4786064 & 0.076357561 & 1.9719262 \\
0.7 & 0.5627976 & 0.5486256 & 0.100331067 & 2.2582014 \\
0.8 & 0.6051595 & 0.6343162 & 0.134119826 & 2.6286980 \\
0.9 & 0.6618487 & 0.7587133 & 0.191882091 & 3.2098076 \\
\hline
\end{tabular}




\subsection{Moments and Conditional Moments}

Let $\mathrm{X} \sim B W L L o G(a, b, c, \alpha, \beta)$, then the $s^{\text {th }}$ moment of $X$ is derived as follows:

$$
E\left(X^{s}\right)=c \sum_{i, j, k=0}^{\infty} w^{*}(i, j, k)(\beta j+\beta+k) \int_{0}^{\infty} x^{s+c-1}\left(1+x^{c}\right)^{-2}\left[1-\left(1+x^{c}\right)^{-1}\right]^{\beta j+\beta+k-1} d x .
$$

Using the binomial series expansion

$$
\left[1-\left(1+x^{c}\right)^{-1}\right]^{\beta j+\beta+k-1}=\sum_{m=0}^{\infty} \frac{(-1)^{m} \Gamma(\beta j+\beta+k)}{m ! \Gamma(\beta j+\beta+k-m)}\left[\left(1+x^{c}\right)^{-m}\right]
$$

and inserting this into the moment of the BWLLoG distribution, we obtain

$$
E\left(X^{s}\right)=c \sum_{i, j, k, m=0}^{\infty} w^{*}(i, j, k) \frac{(-1)^{m} \Gamma(\beta j+\beta+k)}{m ! \Gamma(\beta j+\beta+k-m)}(\beta j+\beta+k) \int_{0}^{\infty} x^{s+c-1}\left[\left(1+x^{c}\right)^{-1}\right]^{(m+2)} d x .
$$

Let $u=\left(1+x^{c}\right)^{-1}$, then $x=\left(\frac{1-u}{u}\right)^{\frac{1}{c}}$ and $d x=-\frac{u^{-2}(1-u)^{\frac{1}{c}-1} u^{1-\frac{1}{c}}}{c} d u$, so that

$$
\begin{aligned}
E\left(X^{s}\right) & =c \sum_{i, j, k, m=0}^{\infty} w^{*}(i, j, k) \frac{(-1)^{m} \Gamma(\beta j+\beta+k)}{m ! \Gamma(\beta j+\beta+k-m)}(\beta j+\beta+k) \int_{0}^{1} u^{m-\frac{s}{c}-\frac{1}{c}-1}(1-u)^{\frac{s}{c}+\frac{1}{c}-1} d u \\
& =c \sum_{i, j, k, m=0}^{\infty} w^{*}(i, j, k) \frac{(-1)^{m} \Gamma(\beta j+\beta+k)}{m ! \Gamma(\beta j+\beta+k-m)}(\beta j+\beta+k) B\left(m-\frac{s}{c}-\frac{1}{c}, \frac{s}{c}+\frac{1}{c}\right),
\end{aligned}
$$

\begin{tabular}{|c|c|c|c|c|}
\hline \multirow[b]{2}{*}{ Moments } & \multicolumn{4}{|c|}{$(a, b, c, \alpha, \beta)$} \\
\hline & $(1.0,2.0,4.0,3.0,2.0)$ & $(1.0,1.0,2.0,4.0,1.0)$ & $(1.0,3.0,1.0,5.0,0.8)$ & $(4.0,3.0,1.0,1.0,0.5)$ \\
\hline $\mathrm{EX}$ & 0.050728788 & 0.4431135 & 0.1275803 & 1.3337918 \\
\hline$E X^{2}$ & 0.031978954 & 0.2500000 & 0.0191371 & 2.8708133 \\
\hline$E X^{3}$ & 0.020788149 & 0.1661675 & 0.0044653 & 7.6577971 \\
\hline$E X^{4}$ & 0.013888884 & 0.1250000 & 0.0014140 & 24.4774222 \\
\hline$E X^{5}$ & 0.009511645 & 0.1038547 & 0.0005656 & 91.5224404 \\
\hline$E X^{6}$ & 0.006662280 & 0.0937500 & 0.0002734 & 393.0969530 \\
\hline $\mathrm{SD}$ & 0.171480448 & 0.2316257 & 0.0534818 & 1.0448984 \\
\hline $\mathrm{CV}$ & 3.380337936 & 0.5227232 & 0.4192009 & 0.7834044 \\
\hline CS & 3.209233007 & 0.6311107 & 8.4587080 & 0.8031220 \\
\hline $\mathrm{CK}$ & 11.732069076 & 3.2450893 & 20.559573 & 4.0017753 \\
\hline
\end{tabular}

where $B(a, b)=\int_{0}^{1} t^{a-1}(1-t)^{b-1} d t$ is the complete beta function. Table 3 lists the first six moments, standard deviation (SD), coefficients of variation (CV), skewness (CS) and kurtosis (CK) of the BWLLoG distribution for selected values of the parameters $(a, b, c, \alpha, \beta)$.

Table 3. The BWLLoG Moments for some selected model parameter values

The $s^{\text {th }}$ conditional moment for the BWLLoG distribution is given as

$$
E\left(X^{s} \mid X>t\right)=\frac{1}{\bar{F}_{B W L L o G}(t)} c \sum_{i, j, k, m=0}^{\infty} w^{*}(i, j, k) \frac{(-1)^{m} \Gamma(\beta j+\beta+k)}{m ! \Gamma(\beta j+\beta+k-m)}(\beta j+\beta+k) B_{\left[1+t^{c}\right]^{-1}}\left(m-\frac{s}{c}-\frac{1}{c}, \frac{s}{c}+\frac{1}{c}\right),
$$

where $B_{u}(a, b)=\int_{0}^{u} t^{a-1}(1-t)^{b-1} d t$ is the incomplete beta function.

5.6 Mean Deviations, Bonferroni and Lorenz Curves

The mean deviation about the mean and the median are expressed as

$$
\delta_{1}(x)=2 \mu F_{B W L L o G}(\mu)-2 \mu+2 T(\mu), \quad \text { and } \quad \delta_{2}(x)=-\mu+2 T(M)
$$


where

$$
\begin{aligned}
T(\mu) & =\int_{\mu}^{\infty} x f_{B W L L o G}(x) d x \\
& =c \sum_{i, j, k, m=0}^{\infty} w^{*}(i, j, k) \frac{(-1)^{m} \Gamma(\beta j+\beta+k)}{m ! \Gamma(\beta j+\beta+k-m)}(\beta j+\beta+k) B_{\left[1+\mu^{c}\right]^{-1}}\left(m-\frac{s}{c}-\frac{1}{c}, \frac{s}{c}+\frac{1}{c}\right) .
\end{aligned}
$$

Bonferroni and Lorenz curves are given as

$$
B(p)=\frac{1}{p \mu} \int_{0}^{q} x f_{B W L L o G}(x) d x=\frac{1}{p \mu}[\mu-T(q)], \quad \text { and } \quad L(p)=\frac{1}{\mu} \int_{0}^{q} x f_{B W L L o G}(x) d x=\frac{1}{\mu}[\mu-T(q)],
$$

where

$$
T(q)=c \sum_{i, j, k, m=0}^{\infty} w^{*}(i, j, k) \frac{(-1)^{m} \Gamma(\beta j+\beta+k)}{m ! \Gamma(\beta j+\beta+k-m)}(\beta j+\beta+k) B_{\left[1+q^{c}\right]^{-1}}\left(m-\frac{s}{c}-\frac{1}{c}, \frac{s}{c}+\frac{1}{c}\right) .
$$

\subsection{Order Statistics}

The density $f_{i: n}(x)$ of the $i^{t h}$ order statistics from the BWLLoG distribution is given as

$$
f_{i: n}(x)=\frac{n !}{(i-1) !(n-i) !} f_{B W L L o G}(x) F_{B W L L o G}^{-1}(x)\left[1-F_{B W L L o G}(x)\right]^{n-i} .
$$

That is,

$$
\begin{aligned}
f_{i: n}(x) & =\sum_{p=0}^{n-i} \frac{n !(-1)^{p}}{(i-1) !(n-i-p) ! p !} f_{B W L L o G}(x)\left[F_{B W L L o G}(x)^{n+i-1}\right] \\
& =\sum_{p=0}^{n-i} \frac{n !(-1)^{p}}{(i-1) !(n-i-p) ! p !} \frac{\alpha \beta c}{B(a, b)} x^{c-1}\left(1+x^{c}\right)^{-2} \frac{\left[1-\left(1+x^{c}\right)^{-1}\right]^{\beta-1}}{\left[\left(1+x^{c}\right)^{-1}\right]^{\beta+1}} \exp \left\{-\alpha b x^{c \beta}\right\} \\
& \times\left[1-\exp \left\{-\alpha x^{c \beta}\right\}\right]^{a-1}\left[I_{1-\exp \left\{-\alpha x^{c \beta}\right\}}(a, b)\right]^{n+i-1} .
\end{aligned}
$$

Using the Binomial series expansion

$$
\left[1-\exp \left\{-\alpha x^{c \beta}\right\}\right]^{a-1}=\sum_{i=0}^{\infty} \frac{(-1)^{i} \Gamma(a)}{i ! \Gamma(a-i)} \exp \left\{-\alpha i x^{c \beta}\right\}
$$

and inserting this back into the pdf of the $i^{\text {th }}$ order statistic from the BWLLoG distribution, we have

$$
\begin{aligned}
f_{i: n}(x) & =\sum_{p=0}^{n-i} \sum_{i=0}^{\infty} \frac{n !(-1)^{i+p} \Gamma(a)}{i ! p !(i-1) !(n-i-p) ! \Gamma(a-i)} \frac{\alpha \beta c}{B(a, b)} x^{c-1}\left(1+x^{c}\right)^{-2} \\
& \times \frac{\left[1-\left(1+x^{c}\right)^{-1}\right]^{\beta-1}}{\left[\left(1+x^{c}\right)^{-1}\right]^{\beta+1}} \exp \left\{-\alpha b x^{c \beta}\right\} \exp \left\{-\alpha i x^{c \beta}\right\}\left[I_{1-\exp \left\{-\alpha x^{c \beta}\right\}}(a, b)\right]^{n+i-1} \\
& =\sum_{p=0}^{n-i} \sum_{i=0}^{\infty} \frac{n !(-1)^{i+p} \Gamma(a)}{i ! p !(i-1) !(n-i-p) ! \Gamma(a-i)} \frac{\alpha \beta c}{B(a, b)} x^{c-1}\left(1+x^{c}\right)^{-2} \\
& \times \frac{\left[1-\left(1+x^{c}\right)^{-1}\right]^{\beta-1}}{\left[\left(1+x^{c}\right)^{-1}\right]^{\beta+1}} \exp \left\{-\alpha(b+i) x^{c \beta}\right\}\left[I_{1-\exp \left\{-\alpha x^{c \beta}\right\}}(a, b)\right]^{n+i-1} .
\end{aligned}
$$

Using the exponential series expansion

$$
\exp \left\{-\alpha(b+i) x^{c \beta}\right\}=\sum_{j=0}^{\infty} \frac{(-1)^{j}[\alpha(b+i)]^{j}}{j !} x^{c \beta j},
$$


and inserting this back into the pdf of the $i^{\text {th }}$ order statistic from the BWLLoG distribution, we have

$$
\begin{aligned}
f_{i: n}(x) & =\sum_{p=0}^{n-i} \sum_{i, j=0}^{\infty} \frac{n !(-1)^{i+j+p}[\alpha(b+i)]^{j} \Gamma(a)}{i ! j ! p !(i-1) !(n-i-p) ! \Gamma(a-i)} \frac{\alpha \beta c}{B(a, b)} x^{c \beta j+c-1}\left(1+x^{c}\right)^{-2} \\
& \times \frac{\left[1-\left(1+x^{c}\right)^{-1}\right]^{\beta-1}}{\left[\left(1+x^{c}\right)^{-1}\right]^{\beta+1}}\left[I_{1-\exp \left\{-\alpha x^{c \beta}\right\}}(a, b)\right]^{n+i-1} \\
& =\sum_{p=0}^{n-i} \sum_{i, j=0}^{\infty} w^{*}(i, j, p) \frac{\left[1-\left(1+x^{c}\right)^{-1}\right]^{\beta-1} x^{c \beta j+c-1}\left(1+x^{c}\right)^{-2}}{\left[\left(1+x^{c}\right)^{-1}\right]^{\beta+1}}\left[I_{1-\exp \left\{-\alpha x^{c \beta}\right\}}(a, b)\right]^{n+i-1},
\end{aligned}
$$

where

$$
w^{* *}(i, j, p)=\frac{n !(-1)^{i+j+p}[\alpha(b+i)]^{j} \Gamma(a)}{i ! j ! p !(i-1) !(n-i-p) ! \Gamma(a-i)} \frac{\alpha \beta c}{B(a, b)} .
$$

\subsection{Rényi Entropy}

Rényi entropy of the BWLLoG distribution is defined as

$$
I_{R}(v)=(1-v)^{-1} \log \left(\int_{-\infty}^{\infty} f_{B W L L o G}^{v}(x) d x\right)
$$

for $v>0$ and $v \neq 1$, that is,

$$
\begin{aligned}
I_{R}(v) & =(1-v)^{-1} \log \left(\frac{(\beta \alpha c)^{v}}{B^{v}(a, b)} \int_{0}^{\infty} x^{c v-v}\left[1+x^{c}\right]^{-2 v} \frac{\left[1-\left(1+x^{c}\right)^{-1}\right]^{\beta v-v}}{\left[\left(1+x^{c}\right)^{-1}\right]^{\beta v+v}}\right. \\
& \left.\times \exp \left\{-\alpha b v x^{c \beta}\right\}\left[1-\exp \left\{-\alpha x^{c \beta}\right\}\right]^{a v-v} d x\right) .
\end{aligned}
$$

Applying the binomial series expansion to obtain the following,

$$
\left[1-\exp \left\{-\alpha x^{c \beta}\right\}\right]^{(a v-v+1)-1}=\sum_{i=0}^{\infty} \frac{(-1)^{i} \Gamma(a v-v+1)}{i ! \Gamma(a v-v-i)} \exp \left\{-\alpha i x^{c \beta}\right\}
$$

and inserting this into Rényi entropy of the BWLLoG distribution in equation (29), we obtain

$$
\begin{aligned}
I_{R}(v) & =(1-v)^{-1} \log \left(\frac{(\beta \alpha c)^{v}}{B^{v}(a, b)} \sum_{i=0}^{\infty} \frac{(-1)^{i} \Gamma(a v-v+1)}{i ! \Gamma(a v-v+1-i)}\right. \\
& \left.\times \int_{0}^{\infty} \frac{x^{c v-v}}{\left[1+x^{c}\right]^{2 v}} \frac{\left[1-\left(1+x^{c}\right)^{-1}\right]^{\beta v-v}}{\left[\left(1+x^{c}\right)^{-1}\right]^{\beta v+v}} \exp \left\{-\alpha(b v+i) x^{c \beta}\right\} d x\right)
\end{aligned}
$$

By using the exponential series expansion,

$$
\exp \left\{-\alpha(b v+i) x^{c \beta}\right\}=\sum_{j=0}^{\infty} \frac{(-1)^{j}[\alpha(b v+i)]^{j}}{j !} x^{c \beta j} .
$$

and inserting this into equation (30), we obtain

$$
\begin{aligned}
I_{R}(v) & =(1-v)^{-1} \log \left(\frac{(\beta \alpha c)^{v}}{B^{v}(a, b)} \sum_{i, j=0}^{\infty} \frac{(-1)^{i+j}[\alpha(b v+i)]^{j} \Gamma(a v-v+1)}{i ! j ! \Gamma(a v-v+1-i)}\right. \\
& \left.\times \int_{0}^{\infty} x^{c v+c \beta j-v}\left[1+x^{c}\right]^{-2 v} \frac{\left[1-\left(1+x^{c}\right)^{-1}\right]^{\beta v-v}}{\left[\left(1+x^{c}\right)^{-1}\right]^{\beta v+v}} d x\right) .
\end{aligned}
$$

Let $u=\left(1+x^{c}\right)^{-1}$, then $x=\left(\frac{1-u}{u}\right)^{\frac{1}{c}}$ and $d x=\frac{u^{-2}(1-u)^{\frac{1}{c}-1} u^{1-\frac{1}{c}}}{c} d u$, so that Rényi entropy for the BWLLoG distribution reduces to

$$
\begin{aligned}
I_{R}(v) & =(1-v)^{-1} \log \left(\frac{(\beta \alpha c)^{v}}{B^{v}(a, b)} \sum_{i, j=0}^{\infty} \frac{(-1)^{i+j}[\alpha(b v+i)]^{j} \Gamma(a v-v+1)}{i ! j ! \Gamma(a v-v+1-i)} \frac{1}{c} \int_{0}^{1} u^{\frac{v}{c}-\beta j-\beta v-\frac{1}{c}-1}(1-u)^{\beta j+\beta v-\frac{v}{c}+\frac{1}{c}-v-1} d u\right) \\
& =(1-v)^{-1} \log \left(\frac{(\beta \alpha c)^{v}}{B^{v}(a, b)} \sum_{i, j=0}^{\infty} \frac{(-1)^{i+j}[\alpha(b v+i)]^{j} \Gamma(a v-v+1)}{i ! j ! \Gamma(a v-v+1-i)} B\left(\frac{v}{c}-\beta j-\beta v-\frac{1}{c}, \beta j+\beta v-\frac{v}{c}+\frac{1}{c}-v\right)\right) .
\end{aligned}
$$




\subsection{Maximum Likelihood Estimation}

The log-likelihood function $\ell(\Delta)=\log (L(\Delta))$ of the BWLLoG distribution is given by

$$
\begin{aligned}
\ell(\Delta) & =n[\ln (\alpha)+\ln (\beta)+\ln (c)-\ln (B(a, b))]+(c-1) \sum_{i=0}^{n} \ln \left(x_{i}\right)-2 \sum_{i=0}^{n} \ln \left(1+x_{i}^{c}\right) \\
& +(\beta-1) \sum_{i=0}^{n}\left[1-\left(1+x_{i}^{c}\right)^{-1}\right]-(\beta+1) \sum_{i=0}^{n}\left[\left(1+x_{i}^{c}\right)^{-1}\right]-\alpha b \sum_{i=0}^{n} x_{i}^{c \beta}+(a-1) \sum_{i=0}^{n} \ln \left[1-\exp \left\{-\alpha x_{i}^{c \beta}\right\}\right] .
\end{aligned}
$$

The partial derivatives of the log-likelihood function with respect to the model parameters are obtained as

$$
\begin{aligned}
& \frac{\partial \ell}{\partial a}=-n[\psi(a)-\psi(a+b)]+\sum_{i=0}^{n}\left[1-\exp \left\{-\alpha x_{i}^{c \beta}\right\}\right], \quad \frac{\partial \ell}{\partial b}=-n[\psi(b)-\psi(a+b)]-\alpha x_{i}^{c \beta}, \\
& \frac{\partial \ell}{\partial c}= \frac{n}{c}+\ln \left(x_{i}\right)+(\beta-1) \sum_{i=0}^{n}\left[\left(1+x_{i}^{c}\right)^{-2}\right] x_{i}^{c} \ln \left(x_{i}\right)-2 \sum_{i=0}^{n} \frac{x_{i}^{c} \ln \left(x_{i}\right)}{\left(1+x_{i}^{c}\right)} \\
&-(a-1) \sum_{i=0}^{n} \frac{\exp \left\{-\alpha x_{i}^{c \beta}\right\} \alpha \beta x_{i}^{c \beta} \ln \left(x_{i}\right)}{\left[1-\exp \left\{-\alpha x_{i}^{c \beta}\right\}\right]}-\alpha \beta b \sum_{i=0}^{n} x_{i}^{c \beta} \ln \left(x_{i}\right)+(\beta+1) \sum_{i=0}^{n} \frac{x_{i}^{c} \ln \left(x_{i}\right)}{\left(1+x_{i}^{c}\right)^{2}}, \\
& \frac{\partial \ell}{\partial \alpha}=\frac{n}{\alpha}+b \sum_{i=0}^{n} x_{i}^{c \beta}-(a-1) \sum_{i=0}^{n}\left[1-\exp \left\{-\alpha x_{i}^{c \beta}\right\}\right]^{-1} \exp \left\{-\alpha x_{i}^{c \beta}\right\} x_{i}^{c \beta},
\end{aligned}
$$

and

$$
\frac{\partial \ell}{\partial \beta}=\frac{n}{\beta}+\sum_{i=0}^{n}\left[1-\left(1+x_{i}^{c}\right)^{-1}\right]-\sum_{i=0}^{\infty}\left(1+x_{i}^{c}\right)^{-1}-\alpha b c \sum_{i=0}^{n} x_{i}^{c \beta} \ln \left(x_{i}\right)-\alpha c(a-1) \sum_{i=0}^{n} \frac{\exp \left\{-\alpha x_{i}^{c \beta}\right\} x_{i}^{c \beta} \ln \left(x_{i}\right)}{\left[1-\exp \left\{-\alpha x_{i}^{c \beta}\right\}\right]}
$$

respectively. The equations obtained by setting the above partial derivatives to zero are not in closed form and the values of the parameters $a, b, c, \alpha, \beta$ must be found by using iterative methods. The maximum likelihood estimates of the parameters, denoted by $\hat{\boldsymbol{\Delta}}$ is obtained by solving the nonlinear equation $\left(\frac{\partial \ell}{\partial a}, \frac{\partial \ell}{\partial b}, \frac{\partial \ell}{\partial c}, \frac{\partial \ell}{\partial \alpha}, \frac{\partial \ell}{\partial \beta}\right)^{T}=\mathbf{0}$, using a numerical method such as Newton-Raphson procedure. The Fisher information matrix is given by $\mathbf{I}(\Delta)=\left[\mathbf{I}_{\theta_{i}, \theta_{j}}\right]_{5 X 5}=E\left(-\frac{\partial^{2} \ell}{\partial \theta_{i} \partial \theta_{j}}\right), i, j=1,2,3,4,5$, can be numerically obtained by MATLAB or R software. The total Fisher information matrix $n \mathbf{I}(\boldsymbol{\Delta})$ can be approximated by

$$
\mathbf{J}(\hat{\boldsymbol{\Delta}}) \approx\left[-\left.\frac{\partial^{2} \ell}{\partial \theta_{i} \partial \theta_{j}}\right|_{\Delta=\hat{\Delta}}\right]_{5 X 5}, \quad i, j=1,2,3,4,5 .
$$

For a given set of observations, the matrix given in equation (32) is obtained after convergence of the Newton-Raphson procedure in MATLAB or R software.

\subsection{Asymptotic Confidence Intervals}

The asymptotic confidence intervals for the parameters of the BWLLoG distribution are presented. Similar results can be obtained for any other models under the class of BWG distributions. Numerically, expectations in the Fisher Information Matrix (FIM) can be obtained. Let $\hat{\boldsymbol{\Delta}}=(\hat{a}, \hat{b}, \hat{c}, \hat{\alpha}, \hat{\beta})$ be the maximum likelihood estimates of $\boldsymbol{\Delta}=(\alpha, \beta, c, a, b)$. Under satisfying conditions for parameters in the interior parameter space, but not on the boundary, we have: $\sqrt{n}(\hat{\Delta}-\Delta) \stackrel{d}{\longrightarrow}$ $N_{5}\left(\underline{0}, I^{-1}(\boldsymbol{\Delta})\right)$, with $I(\boldsymbol{\Delta})$ as the expected Fisher information matrix. Replacing the expected Fisher information matrix with the observed information matrix, the asymptotic behavior remains valid. The multivariate normal distribution $N_{5}\left(\underline{0}, J(\hat{\boldsymbol{\Delta}})^{-1}\right)$, with mean vector $\underline{\mathbf{0}}=(0,0,0,0,0)^{T}$, can be used to construct the confidence intervals and confidence regions for the individual model parameters and for the survival and hazard rate functions. That is, the approximate $100(1-\eta) \%$ two-sided confidence intervals for $\alpha, \beta, c, a$ and $b$ are given by:

$$
\widehat{\alpha} \pm Z_{\frac{\eta}{2}} \sqrt{I_{\alpha \alpha}^{-1}(\hat{\Delta})}, \quad \widehat{\beta} \pm Z_{\frac{\eta}{2}} \sqrt{I_{\beta \beta}^{-1}(\hat{\Delta})}, \quad \widehat{c} \pm Z_{\frac{\eta}{2}} \sqrt{I_{c c}^{-1}(\hat{\Delta})}, \quad \widehat{a} \pm Z_{\frac{\eta}{2}} \sqrt{I_{a a}^{-1}(\hat{\Delta})}, \quad \text { and } \quad \widehat{b} \pm Z_{\frac{\eta}{2}} \sqrt{I_{b b}^{-1}(\hat{\Delta})},
$$

respectively, where $I_{\alpha \alpha}^{-1}(\hat{\boldsymbol{\Delta}}), I_{\beta \beta}^{-1}(\hat{\boldsymbol{\Delta}}), I_{c c}^{-1}(\hat{\boldsymbol{\Delta}}), I_{a a}^{-1}(\hat{\boldsymbol{\Delta}})$ and $I_{b b}^{-1}(\hat{\boldsymbol{\Delta}})$ are the diagonal elements of $I_{n}^{-1}(\hat{\boldsymbol{\Delta}})$, and $Z_{\frac{\eta}{2}}$ is the upper $\frac{\eta}{2} t^{t h}$ percentile of the distribution of the standard normal. 


\subsection{The Likelihood Ratio Test}

The likelihood ratio (LR) test statistic can be used to compare the BWLLoG distribution with its sub-models. The unrestricted estimates $\hat{\alpha}, \hat{\beta}, \hat{c}, \hat{a}$ and $\hat{b}$, and restricted estimates $\tilde{\alpha}, \tilde{\beta}$, and $\tilde{c}$ can be computed in order to construct the LR statistics for testing hypotheses concerning the sub models of the BWLLoG distribution. For example, to test $a=b=1$, (WLLoG against BWLLoG) the LR test statistic reduces to $\omega=2[\ln (L(\hat{\alpha}, \hat{\beta}, \hat{c}, \hat{a}, \hat{b}))-\ln (L(\tilde{\alpha}, \tilde{\beta}, \tilde{c}, 1,1))]$, and the LR test rejects the null hypothesis, when $\omega>\chi_{\epsilon}^{2}$, where $\chi_{\epsilon}^{2}$ denote the upper $100 \epsilon \%$ point of the $\chi^{2}$ distribution with 2 degrees of freedom.

\section{Simulation}

The performance of the BWLLoG distribution is examined by conducting various simulations with different sample sizes $n=50, n=100, n=200, n=400$ and $n=800$. The parameter values are set at: $I: a=1.0, b=1.0, c=1.0, \alpha=0.1, \beta=$ 2.3 and $I I: a=0.9, b=0.9, c=1.0, \alpha=0.1, \beta=2.3$, where each sample is replicated 1000 times. Table 4 lists the mean estimates (MLEs), root mean squared errors (RMSEs) and Average Bias (ABias) of the BWLLoG model parameters. The ABias and RMSE of the MLE $\hat{\Delta}$ are given by

$$
R M S E=\sqrt{\frac{\sum_{i=1}^{N}\left(\hat{\Delta_{i}}-\Delta\right)^{2}}{N}} \text { and } \operatorname{ABias}(\hat{\Delta})=\frac{\sum_{i=1}^{N}\left(\hat{\Delta_{i}}-\Delta\right)}{N},
$$

respectively. According to the simulation results given in Table 4, as the sample size $n$ increases, the RMSEs decay towards zero, the mean estimates tend to be closer to the true parameter values and biases decreases overall, however for the parameter $b$, the mean mean estimate fluctuates around the true parameter values and the average biases are very small and negative.

\section{Application}

In this section, a dataset is used to compare the fit of the BWLLoG distribution with three sub-models: WLLoG, ELLoG, RLLoG distributions and the non-nested beta Weibull Poisson (BWP) distribution (Percontini, Blas \& Cordeiro (2013)). The data set used is taken from Shanker, Fasshaye \& Selvaraj (2015), correspond to the time-to-failure of a polyester/viscose yarn in a textile experiment for testing the tensile fatigue characteristics of yarn. It consists of a sample of $100 \mathrm{~cm}$ yarn at $2.3 \%$ strain level. The initial values that were used in $\mathrm{R}$ software to get the estimates are $a=2.0, b=2.0, c=0.01, \alpha=3.0$ and $\beta=1.0$. The estimated values of the model parameters (standard error in parenthesis), -2log-likelihood statistic, Akaike Information Criterion, $A I C=2 p-2 \ln (L)$ Bayesian Information Criterion, $B I C=p \ln (n)-2 \ln (L)$, where $L=L(\hat{\Delta})$ is the value of the likelihood function evaluated at the parameter estimates, $n$ is the number of observations, and $p$ is the number of estimated parameters are presented in Table 6. The goodness-offit statistics (Cramér-von Mises statistic $W^{*}$ ) and (Anderson-Darling statistic $A^{*}$ ), as well as Kolmogorov Smirnov (KS) statistics are also presented in the table. These statistics can be used to verify which distribution fits better to the data. In general, the smaller the values of $W^{*}$ and $A^{*}$, the better the fit. The AdequacyModel package was used to evaluate the statistics stated above.

The estimated asymptotic covariance matrix of the MLE's which is the inverse of the Fisher Information Matrix (FIM) $\mathbf{I}_{n}^{-1}(\hat{\boldsymbol{\Delta}})$, is given by:

$$
\left(\begin{array}{ccccc}
0.001179 & 0.0002159 & 0.0000126 & -0.00001384 & 0.0001996 \\
0.0002156 & 0.00003954 & 0.00000231 & -0.00000253 & 0.0000366 \\
0.0000126 & 0.00000231 & 0.000000135 & -0.000000148 & 0.00000213 \\
-0.00001384 & -0.00000253 & -0.000000148 & 0.000000166 & -0.00000234 \\
0.0001996 & 0.0000366 & 0.00000213 & -0.00000234 & 0.0000338
\end{array}\right),
$$

and the $95 \%$ asymptotic confidence intervals for the parameters are given by: $a \in(0.47630167 \pm 1.96 \times 0.03408149), b \in$ $(1.36232254 \pm 1.96 \times 0.00618560), c \in(0.97617533 \pm 1.96 \times 0.00036084), \alpha \in(0.00070914 \pm 1.96 \times 0.00040809), \beta \in$ $(2.86455102 \pm 1.96 \times 0.00566690)$, respectively.

The likelihood ratio (LR) statistics for testing the hypothesis $H_{0}$ : WLLoG versus $H_{a}$ : BWLLoG, $H_{0}$ : ELLoG versus $H_{a}$ : BWLLoG, and $H_{0}$ : RLLoG versus $H_{a}$ : BWLLoG are 28.223 (p-value $\left.<0.0001\right), 17.131$ (p-value $=0.0007<0.01$ ), and 157.176 (p-value $<0.0001$ ), respectively. In all the three LR tests, we reject the null hypothesis and conclude that there are significant differences between the BWLLoG and each of the nested WLLoG, ELLoG, RLLoG distributions.

The fitted densities given in Figure 5 shows that the BWLLoG distribution is a very good fit for the yarn data. The statistics: AIC and BIC as well as the goodness-of-fit statistics $W^{*}$ and $A^{*}$ clearly show that the BWLLoG distribution is a better fit than the nested WLLoG, ELLoG, and RLLoG distributions and non-nested BWP distribution for the Yarn data set. 
Table 4. Monte Carlo Simulation Results: Mean Estimates, ABias and RMSEs

\begin{tabular}{|c|c|c|c|c|c|c|c|}
\hline \multirow[b]{2}{*}{ Parameter } & \multirow[b]{2}{*}{$\mathrm{n}$} & \multicolumn{3}{|c|}{ I } & \multicolumn{2}{|c|}{ II } & \multirow[b]{2}{*}{ RMSE } \\
\hline & & Mean & ABias & RMSE & Mean & ABias & \\
\hline \multirow[t]{5}{*}{$a$} & 50 & 1.3011 & 0.3011 & 4.9600 & 1.1195 & 0.2195 & 2.9325 \\
\hline & 100 & 0.9979 & -0.0021 & 0.4688 & 0.9325 & 0.0325 & 0.4671 \\
\hline & 200 & 0.9924 & -0.0076 & 0.3121 & 0.9101 & 0.0101 & 0.2837 \\
\hline & 400 & 0.9859 & -0.0141 & 0.2327 & 0.8913 & -0.0087 & 0.2016 \\
\hline & 800 & 0.9866 & -0.0134 & 0.1658 & 0.8911 & -0.0089 & 0.1418 \\
\hline \multirow[t]{5}{*}{$b$} & 50 & 0.9217 & -0.0783 & 2.8513 & 0.8380 & -0.0620 & 1.1623 \\
\hline & 100 & 0.8710 & -0.1290 & 0.6001 & 0.8565 & -0.0435 & 0.5630 \\
\hline & 200 & 0.8897 & -0.1103 & 0.5631 & 0.8964 & -0.0036 & 0.5630 \\
\hline & 400 & 0.8769 & -0.1231 & 0.5521 & 0.8651 & -0.0349 & 0.5220 \\
\hline & 800 & 0.9006 & -0.0994 & 0.4913 & 0.8635 & -0.0365 & 0.4671 \\
\hline \multirow[t]{5}{*}{$c$} & 50 & 1.0920 & 0.0920 & 0.2212 & 1.0883 & 0.0883 & 0.2170 \\
\hline & 100 & 1.0553 & 0.0553 & 0.1585 & 1.0480 & 0.0480 & 0.1631 \\
\hline & 200 & 1.0313 & 0.0313 & 0.1133 & 1.0241 & 0.0241 & 0.1148 \\
\hline & 400 & 1.0220 & 0.0220 & 0.0847 & 1.0184 & 0.0184 & 0.0855 \\
\hline & 800 & 1.0140 & 0.0140 & 0.0661 & 1.0113 & 0.0113 & 0.0643 \\
\hline \multirow[t]{5}{*}{$\alpha$} & 50 & 0.2774 & 0.1774 & 0.8214 & 0.3438 & 0.2438 & 1.2298 \\
\hline & 100 & 0.2474 & 0.1474 & 0.6991 & 0.3183 & 0.2183 & 1.6994 \\
\hline & 200 & 0.2170 & 0.1170 & 0.5640 & 0.2233 & 0.1233 & 0.5508 \\
\hline & 400 & 0.1944 & 0.0944 & 0.2489 & 0.2035 & 0.1035 & 0.3668 \\
\hline & 800 & 0.1682 & 0.0682 & 0.2303 & 0.1943 & 0.0943 & 0.3686 \\
\hline \multirow[t]{5}{*}{$\beta$} & 50 & 2.4333 & 0.1333 & 0.3834 & 2.4232 & 0.1232 & 0.3783 \\
\hline & 100 & 2.3801 & 0.0801 & 0.2525 & 2.3616 & 0.0616 & 0.2621 \\
\hline & 200 & 2.3432 & 0.0432 & 0.1721 & 2.3314 & 0.0314 & 0.1617 \\
\hline & 400 & 2.3283 & 0.0283 & 0.1206 & 2.3218 & 0.0218 & 0.1151 \\
\hline & 800 & 2.3164 & 0.0164 & 0.0860 & 2.3071 & 0.0071 & 0.0799 \\
\hline
\end{tabular}

Table 5. Yarn Dataset at 2.3\% Strain Level

\begin{tabular}{cccccccccc}
\hline 86 & 146 & 251 & 653 & 98 & 249 & 400 & 292 & 131 & 169 \\
175 & 176 & 76 & 264 & 15 & 364 & 195 & 262 & 88 & 264 \\
157 & 220 & 42 & 321 & 180 & 198 & 38 & 20 & 61 & 121 \\
282 & 224 & 149 & 180 & 325 & 250 & 196 & 90 & 229 & 166 \\
38 & 337 & 65 & 151 & 341 & 40 & 40 & 135 & 597 & 246 \\
211 & 180 & 93 & 315 & 353 & 571 & 124 & 279 & 81 & 186 \\
497 & 182 & 423 & 185 & 229 & 400 & 338 & 290 & 398 & 71 \\
246 & 185 & 188 & 568 & 55 & 55 & 61 & 244 & 20 & 284 \\
393 & 396 & 203 & 829 & 239 & 236 & 286 & 194 & 277 & 143 \\
198 & 264 & 105 & 203 & 124 & 137 & 135 & 350 & 193 & 188 \\
\hline
\end{tabular}


Table 6. Estimation of BWLLoG model for Yarn Dataset

\begin{tabular}{|c|c|c|c|c|c|c|c|c|c|c|c|}
\hline \multirow[b]{2}{*}{ Model } & \multicolumn{3}{|c|}{ Estimates } & \multicolumn{4}{|c|}{ Statistics } & \multirow[b]{2}{*}{$A^{*}$} & \multirow[b]{2}{*}{$A I C$} & \multirow[b]{2}{*}{$B I C$} & \multirow[b]{2}{*}{$K S$} \\
\hline & $\hat{a}$ & $\hat{b}$ & $\hat{c}$ & $\hat{\alpha}$ & $\hat{\hat{\beta}}$ & $-2 \log L$ & $W^{*}$ & & & & \\
\hline \multirow[t]{2}{*}{ BWLLOG } & 0.47630167 & 1.36232254 & 0.97617533 & 0.00070914 & 2.86455102 & 1249.825 & 0.09596863 & 0.5397216 & 1259.825 & 1272.8511 & 0.082306 \\
\hline & $(0.03408149)$ & $(0.00618560)$ & $(0.00036084)$ & $(0.00040809)$ & $(0.005666090)$ & & & & & & \\
\hline \multirow[t]{2}{*}{ WLLOG } & - & - & 0.5343284 & 0.0040360 & 1.9143012 & 1278.048 & 0.1219875 & 0.6650875 & 1284.048 & 1291.864 & 0.20013 \\
\hline & - & - & $(0.0680358)$ & $(0.0032991)$ & $(0.2437472)$ & & & & & & \\
\hline \multirow[t]{2}{*}{ ELLoG } & - & - & 1.1446091 & 0.0020622 & - & 1266.956 & 0.108994 & 0.6008626 & 1270.956 & 1276.167 & 0.17309 \\
\hline & - & - & $(0.6466751)$ & $(0.0077794)$ & - & & & & & & \\
\hline \multirow[t]{3}{*}{ RLLOG } & - & - & 0.5632383 & 0.0022730 & - & 1407.001 & 0.110811 & 0.6096597 & 1411.001 & 1416.211 & 0.17616 \\
\hline & - & - & $(0.1051231)$ & $(0.0027792)$ & - & & & & & & \\
\hline & $\hat{a}$ & $\hat{b}$ & $\hat{a}$ & $\hat{\beta}$ & $\hat{\imath}$ & & & & & & \\
\hline \multirow[t]{2}{*}{ BWP } & 1.34424491 & 0.89290911 & 1.38113954 & 0.00069060 & 0.02442390 & 1254.732 & 0.09608055 & 0.5406132 & 1264.732 & 1277.758 & 0.082144 \\
\hline & $(0.5730770)$ & $(0.0877171)$ & $(0.2068545)$ & $(0.00186668)$ & $(0.0182989)$ & & & & & & \\
\hline
\end{tabular}

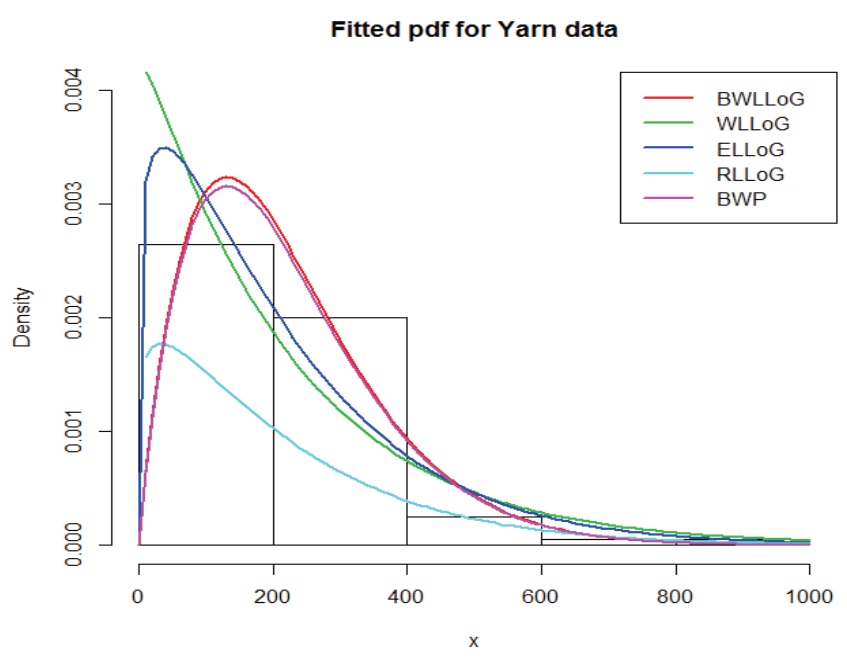

Figure 5. Fitted pdf plot of the BWLLoG distribution for Yarn dataset

\section{Concluding Remarks}

A new generalization of the Weibull distribution, namely the beta Weibull-G family of distributions was introduced and studied. Statistical properties of the BWG family such as expansion of density function, hazard and reverse hazard functions, quantile function, moments, incomplete moments, mean deviations, Bonferroni and Lorenz curves, order statistics, Rényi entropy and maximum likelihood estimation for the parameters were developed. Also, three special cases of the BWG family of distributions were discussed, namely the BWU, BWE and BWLLoG distributions along with an application to real life data. The application of the special case of the BWLLoG distribution show that it perform better than the nested distributions and the non-nested BWP distribution.

\section{Acknowledgements}

The authors would like to thank the editor and the referees for carefully reading the paper and for comments which greatly improved the presentation in this paper.

\section{References}

Almalki, S. J., \& Nadarajah, S. (2015). Comparing the Exponentiated and Generalized Modified Weibull Distributions. Journal of Data Science, 13, 713-732.

Barreto-Souza, W., Santos, A. H. S., \& Cordeiro, G. M. (2010). The Beta Generalized Exponential Distribution. Journal 
of Statistical Computation and Simulation, 80, 159-172. https://doi.org/10.1080/00949650802552402

Bourguignon, M., Silva, R. S., \& Cordeiro, G. M. (2014). The Weibull-G Family of Distributions. Journal of Data Science, $12,53-68$.

Carrasco, M., Cordeiro, G. M., \& Ortega, E. M. (2008). A Generalized Modified Weibull Distribution for Lifetime Modeling. Computational Statistics and Data Analysis, 53(2), 450-462. https://doi.org/10.1016/j.csda.2008.08.023

Cordeiro, G. M., da Cunha, D. C. C., \& Ortega, E. M. M. (2013). The Exponentiated Generalized Class of Distributions. Journal of Data Science, 11, 1-27.

Elbatal, I., \& Merovci, F. (2015). A New Generalized of Exponentiated Modified Weibull Distribution. Journal of Data Science, 13, 213-240.

Eugene, N., Famoye, F., \& Lee, C. (2002). Beta-Normal Distribution and Its Applications. Communications in Statistics: Theory and Methods, 31, 497-512. https://doi.org/10.1081/STA-120003130

Gurvich, M. R., DiBenedetto, A. T., \& Ranade, S. V. (1997). A New Statistical Distribution for Characterizing the Random Strength of Brittle Materials. Journal of Materials Science, 32, 2559-2564. https://doi.org/10.1023/A:1018594215963

Lee, C., Famoye, F., \& Olumolade, O. (2007). Beta-Weibull Distribution: Some Properties and Applications. Journal of Modern Applied Statistical Methods, 6, 173-186. https://doi.org/10.22237/jmasm/1177992960

Nadarajah, S., \& Gupta, A. K. (2004). The Beta Frechet Distribution. Far East Journal of Theoretical Statistics, 14, 15-24.

Nadarajah, S., \& Kotz, S. (2004). The Beta Gumbel Distribution. Mathematical Problems in Engineering, 10, $323-332$. https://doi.org/10.1155/S1024123X04403068

Nadarajah, S., \& Kotz, S. (2005). The Beta exponential Distribution. Reliability Engineering and System Safety, 91, 689-697. https://doi.org/10.1016/j.ress.2005.05.008

Oluyede, B. O., \& Yang, T. (2015). A New Class of Generalized Lindley Distributions with Applications. Journal of Statistical Computation and Simulation, 85(10), 2072-2100. https://doi.org/10.1080/00949655.2014.917308

Oluyede, B. O., Huang, S., \& Yang, T. (2015). A New Class of Generalized Modified Weibull Distributions with Applications. Austrian Journal of Statistics, 44, 45-68. https://doi.org/10.17713/ajs.v44i3.36

Oluyede, B. O., Pu, S., Makubate, B., \& Qiu, Y. (2018). The Gamma Weibull-G Family of Distributions, To appear in Austrian Journal of Statistics.

Percontini, A., Blas, B., \& Cordeiro, G. M. (2013). The Beta Weibull Poisson Distribution. Chilean Journal of Statistics, 4(2), 3-26.

Pescim, R. R., Demetrio, C. G. B., Cordeiro, G. M., Ortega, E. M. M., \& Urbano, M. R. (2010). The Beta Generalized Half-Normal Distribution. Computational Statistics and Data Analysis, 54, 945-957. https://doi.org/10.1016/j.csda.2009.10.007

Shanker, R., Fesshaye, H., \& Selvaraj, S. (2015). On Modeling of Lifetimes Data Using Exponential and Lindley Distributions. Biometrics and Biostatistics International Journal, 2(5), 4-9.

Tahir, M. H., Zubair, M., Mansoor, M., Cordeiro, G. M., Alizadeh, M., \& Hamedani, G. G. (2016). A New Weibull-G Family of Distributions. Hacettepe Journal of Mathematics and Statistics, 45(2), 629-647.

Zografos, K., \& Balakrishnan, N. (2009). Families of Beta- and Generalized Gamma-Generated Distribution and Associated Inference, Statistical Methodology, 6, 344-362. https://doi.org/10.1016/j.stamet.2008.12.003

\section{Copyrights}

Copyright for this article is retained by the author(s), with first publication rights granted to the journal.

This is an open-access article distributed under the terms and conditions of the Creative Commons Attribution license (http://creativecommons.org/licenses/by/4.0/). 